Preprint: ZU-TH-30/95, hep-th/9606105

\title{
Slavnov-Taylor Identities from the Causal Point of View
}

\author{
Michael Dütsch \\ Institut für Theoretische Physik der Universität Zürich \\ Winterthurerstr. 190, CH-8057 Zürich, Switzerland
}

\begin{abstract}
We continue the investigation of quantized Yang-Mills theories coupled to matter fields in the framework of causal perturbation theory which goes back to Epstein and Glaser. In this approach gauge invariance is expressed by a simple commutator relation for the S-matrix and the corresponding gauge transformations are simple transformations of the free fields only. In spite of this simplicity, gauge invariance implies the usual SlavnovTaylor identities. The main purpose of this paper is to prove the latter statement. Since the Slavnov-Taylor identities are formulated in terms of Green's functions, we investigate the agreement of two perturbative definitions of Green's functions, namely of Epstein and Glaser's definition with the Gell-Mann Low series.
\end{abstract}

PACS. 11.10 - Field theory, 12.35C-General properties of quantum chromodynamics. 


\section{Introduction}

\section{(a) The Model}

In a recent series of papers $[1,2,3,4,5]$ non-abelian gauge invariance has been studied in the framework of causal perturbation theory. This approach, which goes back to Epstein and Glaser [6], has the merit that one works exclusively with free fields, which are mathematically well-defined, and performs only justified operations with them. Consequently, the gauge transformations are transformations of the free fields only.

In causal perturbation theory one makes an ansatz for the S-matrix as a formal power series in the coupling constant

$$
S(g)=1+\sum_{n=1}^{\infty} \frac{1}{n !} \int d^{4} x_{1} \ldots d^{4} x_{n} T_{n}\left(x_{1}, \ldots, x_{n}\right) g\left(x_{1}\right) \ldots g\left(x_{n}\right)
$$

The test function $g \in \mathcal{S}\left(\mathbf{R}^{4}\right)$ switches the interaction and $T_{n}\left(x_{1}, \ldots, x_{n}\right)$ is an operator-valued distribution. The $T_{n}$ 's are constructed inductively from the given first order

$$
T_{1}(x)=T_{1}^{A}(x)+T_{1}^{u}(x)+T_{1}^{\psi}(x)
$$

with

$$
\begin{gathered}
T_{1}^{A}(x) \stackrel{\text { def }}{=} \frac{i g}{2} f_{a b c}: A_{\mu a}(x) A_{\nu b}(x) F_{c}^{\nu \mu}(x):, \\
T_{1}^{u}(x) \stackrel{\text { def }}{=}-i g f_{a b c}: A_{\mu a}(x) u_{b}(x) \partial^{\mu} \tilde{u}_{c}(x):, \\
T_{1}^{\psi}(x) \stackrel{\text { def }}{=} i j_{\mu a}(x) A_{a}^{\mu}(x),
\end{gathered}
$$

where the matter current $j_{\mu a}$ is defined by

$$
j_{\mu a}(x) \stackrel{\text { def }}{=} \frac{g}{2}: \bar{\psi}_{\alpha}(x) \gamma_{\mu}\left(\lambda_{a}\right)_{\alpha \beta} \psi_{\beta}(x): \text {. }
$$

Herein, $g$ is the coupling constant, $f_{a b c}$ are the structure constants of the group SU(N) and $\frac{-i}{2} \lambda_{a}, a=1, \ldots, N^{2}-1$ denote the generators of the fundamental representation of $\mathrm{SU}(\mathrm{N})$. The gauge potentials $A_{a}^{\mu}, F_{a}^{\mu \nu} \stackrel{\text { def }}{=} \partial^{\mu} A_{a}^{\nu}-\partial^{\nu} A_{a}^{\mu}$, and the ghost fields $u_{a}, \tilde{u}_{a}$ are massless and fulfil the wave equation. The matter fields $\psi_{\alpha}$ and $\bar{\psi}_{\alpha} \stackrel{\text { def }}{=} \psi_{\alpha}^{+} \gamma^{0}$ satisfy the free Dirac equation with a colour independent mass $m \geq 0$ [5]. Therefore, the matter current is conserved

$$
\partial^{\mu} j_{\mu a}(x)=0
$$

Gauge invariance means roughly speaking that the commutator of the $T_{n}$-distributions with the gauge charge

$$
Q \stackrel{\text { def }}{=} \int_{t=\text { const. }} d^{3} x\left(\partial_{\nu} A_{a}^{\nu} \stackrel{\leftrightarrow}{\partial}_{0} u_{a}\right)
$$

is a (sum of) divergence(s). In first order this holds true

$$
\left[Q, T_{1}^{A}(x)+T_{1}^{u}(x)\right]=i \partial_{\nu}\left(T_{1 / 1}^{A \nu}(x)+T_{1 / 1}^{u \nu}(x)\right)
$$


where

$$
\begin{gathered}
T_{1 / 1}^{A \nu}(x) \stackrel{\text { def }}{=} i g f_{a b c}: A_{\mu a}(x) u_{b}(x) F_{c}^{\nu \mu}(x):, \\
T_{1 / 1}^{u \nu}(x) \stackrel{\text { def }}{=}-\frac{i g}{2} f_{a b c}: u_{a}(x) u_{b}(x) \partial^{\nu} \tilde{u}_{c}(x):
\end{gathered}
$$

and, by means of the current conservation (1.7),

$$
\left[Q, T_{1}^{\psi}(x)\right]=i \partial_{\nu} T_{1 / 1}^{\psi \nu}(x)
$$

with

$$
T_{1 / 1}^{\psi \nu}(x) \stackrel{\text { def }}{=} i j_{a}^{\nu}(x) u_{a}(x)
$$

Note that $\left[Q, T_{1}^{A}\right]$ alone is not a divergence. In order to have gauge invariance in first order, we are forced to introduce the ghost coupling $T_{1}^{u}(1.4)$. We define gauge invariance in arbitrary order by

$$
\left[Q, T_{n}\left(x_{1}, \ldots, x_{n}\right)\right]=i \sum_{l=1}^{n} \partial_{\mu}^{x_{l}} T_{n / l}^{\mu}\left(x_{1}, \ldots, x_{n}\right)
$$

The divergences on the r.h.s. of (1.14) are given by $n$-th order $T$-distributions from a different theory which contains, in addition to the usual Yang-Mills couplings (1.2), a socalled Q-vertex, defined by $T_{1 / 1}^{\nu}=T_{1 / 1}^{A \nu}+T_{1 / 1}^{u \nu}+T_{1 / 1}^{\psi \nu}(1.10),(1.11)$, (1.13). (See ref. [2] for more details.) Gauge invariance (1.14) implies the invariance of the S-matrix (1.1) with respect to simple gauge transformations of the free fields [5]. These transformations are the free field version of the famous BRS-transformations [7]. Moreover, unitarity on the physical subspace can be proven by means of gauge invariance (1.14) [4].

As usual, the $T_{n}$ 's are constructed in normally ordered form

$$
T_{n(/ l)}\left(x_{1}, \ldots, x_{n}\right)=\sum_{\mathcal{O}} t_{\mathcal{O}}^{(l)}\left(x_{1}-x_{n}, \ldots, x_{n-1}-x_{n}\right): \mathcal{O}\left(x_{1}, \ldots, x_{n}\right):
$$

where $\mathcal{O}\left(x_{1}, \ldots, x_{n}\right)$ is a combination of free field operators and $t_{\mathcal{O}}^{(l)}$ is a C-number distribution. The latter contains an undetermined but finite normalization polynomial

$$
t_{\mathcal{O}}^{(l)}\left(x_{1}-x_{n}, \ldots\right)+\sum_{|a|=0}^{\omega(\mathcal{O})} C_{a} D^{a} \delta^{(4(n-1))}\left(x_{1}-x_{n}, \ldots, x_{n-1}-x_{n}\right)
$$

of degree $\omega(\mathcal{O})$. One can prove $[2,8]$

$$
\omega(\mathcal{O})=4-b-g-d-\frac{3}{2} f
$$

where $b$ is the number of gauge bosons $(A, F), g$ the number of ghosts $(u, \partial \tilde{u}), d$ the number of derivatives $(F, \partial \tilde{u})$ and $f$ is the number of pairs $(\bar{\psi}, \psi)$ in $\mathcal{O}$. The fact that $\omega$ is bounded in the order $n$ (here it is even independent on $n$ ) is the (re)normalizability of this model.

The constants $C_{a}$ in (1.16) are restricted by symmetry properties, especially by gauge invariance. The most important example is the second order tree diagram $b=4, g=f=$ $d=0$, which has the normalization term

$$
C i g^{2}: A_{\mu a}\left(x_{1}\right) A_{\nu b}\left(x_{1}\right) A_{d}^{\mu}\left(x_{2}\right) A_{e}^{\nu}\left(x_{2}\right): f_{a b c} f_{d e c} \delta\left(x_{1}-x_{2}\right)
$$


Gauge invariance (1.14) fixes the value of $C$ uniquely: $C=-\frac{1}{2}$ [1,5]. With this value, (1.18) agrees with the usual four-gluon coupling. It propagates to higher orders in the inductive construction of the $T_{n}$ 's (sect. 4.2 of [9]).

\section{(b) The Aim of the Paper}

The operator gauge invariance (1.14) can be expressed by the Cg-identities, the Cnumber identities for gauge invariance, which imply (1.14). The Cg-identities have been proven by induction on the order $n$ in $[2,3,4,5]$. However, they still contain the Q-vertex, which is only a mathematical auxiliary tool to formulate gauge invariance (1.14). Our first goal will be to eliminate the $Q$-vertex from the $C g$-identities. This is done in sect.2 by inserting the $\mathrm{Cg}$-identities into each other and taking the limit of vanishing inner momenta. We call the resulting equations reduced Cg-identities. Moreover, we study the (finite) renormalizations (1.16) preserving the latter identities rsp. the original Cg-identities.

As mentioned above our gauge transformations in the perturbative S-matrix (1.1) involve free fields only. Especially a local transformation of the matter fields is excluded, since $e^{i \Lambda(x)} \psi_{\alpha}(x)$ does not fulfil the free Dirac equation. One may ask, therefore, whether (1.14) contains the full information of non-abelian gauge invariance. It is the aim of this paper to prove this, apart from the independence on the gauge fixing, which is not considered here. (We always work in Feynman gauge.) The usual gauge or BRS invariance is expressed by the famous Slavnov-Taylor identities [10,11,12]. In sect.3 we prove the latter in the framework of perturbation theory by means of our reduced Cg-identities. There is a interesting speciality concerning matter fields: For the Cg-identities with a pair $(\bar{\psi}, \psi)$ among the external legs, the Q-vertex cannot be eliminated completely. But in this case also Taylor [10] was forced to introduce the $Q$-vertex $T_{1 / 1}^{\psi}(1.13)$ to formulate his identities.

The Slavnov-Taylor identities are written in terms of connected or in terms of oneparticle irreducible Green's functions. The latter cannot be expressed directly by our $t_{\mathcal{O}^{-}}$ distributions (1.15), since in higher orders the $t_{\mathcal{O}}$ 's contain one-particle reducible terms. We must go another way: We work with the Gell-Mann Low series [13] for the connected Green's functions. Epstein and Glaser [6] have given another definition of Green's functions in the framework of causal perturbation theory. In the appendix we prove that their definition agrees with the Gell-Mann Low expressions at least for massive theories.

\section{(c) Notations}

We recall our convention given in [2-5] of denoting numerical distributions of nondegenerate terms, i.e. connected terms with at most one external field operator at each vertex. The expression

$$
t_{A B \ldots a b \ldots}^{\alpha 2}\left(x_{2}, x_{3}, \ldots\right): A_{a}\left(x_{2}\right) B_{b}\left(x_{3}\right) \ldots:
$$

means the sum of all connected diagrams with external field operators (legs) $A_{a}\left(x_{2}\right)$, $B_{b}\left(x_{3}\right) \ldots, a$ and $b$ are colour indices. (We only write the index $\tilde{u}$ instead of $\partial \tilde{u}$ for an external field operator $\partial_{\nu} \tilde{u}\left(x_{i}\right)$.) The subscripts $\alpha 2$ show that this term belongs to $T_{n / 3}^{\alpha}\left(x_{1}, x_{2}, x_{3}, \ldots\right)$ with $Q$-vertex at the second argument $\left(=x_{3}\right)$ of the numerical distribution $t$. An immediate consequence of this notation is the relation

$$
t_{A B \ldots a b \ldots}^{\alpha 1}\left(x_{1}, x_{2}, \cdots\right)= \pm t_{B A \ldots b a \ldots}^{\alpha 2}\left(x_{2}, x_{1}, \cdots\right)
$$


where we have a minus sign, if $A, B$ are both ghost or both matter operators and a plus sign in all other cases. The Lorentz indices of the two operators $A, B$ must also be reversed. Note that the latter equation particularly holds, if $A$ and $B$ are the same field operators. Moreover, the sum over permutations of the vertices is present in $T_{n(/ l)}$. For example,

$$
\begin{gathered}
T_{n}\left(x_{1}, \ldots x_{n}\right)=\sum_{i, j, k, l=1}^{n}: \bar{\psi}\left(x_{i}\right) t_{\bar{\psi} \psi A A a b}^{\mu \nu}\left(x_{i}, x_{j}, x_{k}, x_{l}, x_{1} \ldots \bar{x}_{i}, \bar{x}_{j}, \bar{x}_{k}, \bar{x}_{l} \ldots, x_{n}\right) . \\
\cdot \psi\left(x_{j}\right) A_{\mu a}\left(x_{k}\right) A_{\nu b}\left(x_{l}\right):+\ldots+(\text { disconnected terms }),
\end{gathered}
$$

where the coordinates with bar must be omitted and the sum runs only over the region $i \neq j, i \neq k, i \neq l, j \neq k, j \neq l$ and $k<l$. The latter restriction is not $k \neq l$, since the external field operators at the vertices $x_{k}$ and $x_{l}$ agree. The matrix multiplication : $\bar{\psi} t(\ldots) \psi:$ concerns the spinor space and the space of the fundamental representation. We emphasize that in higher orders our $t$-distributions contain one-particle reducible terms.

The degenerate terms have at least one vertex with two external legs. We shall write them in terms of non-degenerate $t$-distributions of lower orders and Feynman propagators.

Some numerical distributions can be factorized $[3,5]$ concerning their Lorentz structure

$$
\begin{aligned}
& t_{u u \tilde{u} \ldots}^{\nu 3 \mu}=g^{\nu \mu} \vec{t}_{u u \tilde{u} \ldots}^{3},
\end{aligned}
$$

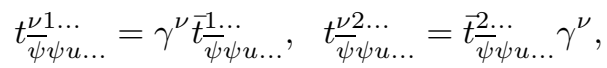

or concerning their colour structure

$$
\begin{gathered}
t_{B_{1} B_{2} a b}=\delta_{a b} t_{B_{1} B_{2}}^{\prime}, \quad t_{B_{1} B_{2} B_{3} a b c}=f_{a b c} t_{B_{1} B_{2} B_{3}}^{\prime}, \\
t_{\bar{\psi} \psi \alpha \beta}=\tau_{\bar{\psi} \psi} \delta_{\alpha \beta}, \quad t_{\bar{\psi} \psi B a \alpha \beta}=\tau_{\bar{\psi} \psi B}\left(\lambda_{a}\right)_{\alpha \beta},
\end{gathered}
$$

where $B, B_{1}, B_{2}, B_{3} \in\{A, F, u, \tilde{u}\}$ and $a, b, c$ (rsp. $\left.\alpha, \beta\right)$ are indices of the adjoint (rsp. fundamental) representation of $\mathrm{SU}(\mathrm{N})$. We shall omit the prime in $t_{B_{1} B_{2}\left(B_{3}\right)}^{\prime}$. Due to translation invariance, the numerical distributions depend on the relative coordinates $\left(x_{1}-x_{n}, x_{2}-\right.$ $\left.x_{n}, \ldots, x_{n-1}-x_{n}\right)$ only. The Fourier transformation is done in the latter coordinates and the hat in $\hat{t}\left(p_{1}, p_{2}, \ldots, p_{n-1}\right)$ is mostly omitted.

\section{(d) Assumption about the Infrared Behaviour}

In the present paper we work with the following assumption throughout: Let $p_{1}, \ldots, p_{r}$ be the external and $p_{r+1}, \ldots, p_{n-1}$ be the inner momenta of an arbitrary $\hat{t}$-distribution without or with one $\mathrm{Q}$-vertex. Moreover we set $p_{n} \stackrel{\text { def }}{=}-\left(p_{1}+p_{2}+\ldots+p_{n-1}\right)$. We assume that for

$$
p_{r+1}, \ldots, p_{n-1}, p_{n} \rightarrow 0, \text { and for all } p_{1}, \ldots, p_{r-1} \text { and } \tilde{p}_{r} \stackrel{\text { def }}{=}-\left(p_{1}+\ldots+p_{r-1}\right) \text { off }- \text { shell }
$$

the limit of the considered $\hat{t}$-distribution exists

$$
\lim _{p_{r+1}, \ldots, p_{n-1}, p_{n} \rightarrow 0} \hat{t}\left(p_{1}, p_{2}, \ldots, p_{n-1}\right)=\left.\hat{t}\left(p_{1}, \ldots, p_{r}, 0, \ldots 0\right)\right|_{p_{1}+\ldots+p_{r}=0}
$$

The mass-shell is meant to be the set $\left\{p \in \mathbf{R}^{4} \mid p^{2}=m^{2}\right\}$. It is absolutely necessary that the external momenta are off-shell because otherwise infrared divergences appear (even in QED $[14,15])$ and the limit does not exist. 
Remarks: (1) The vacuum diagrams $(r=0)$ are excluded in (1.25). At the end of the appendix it is shown that they really violate this assumption.

(2) The limit (1.24-25) is understood in the following sense: We start in $x$-space and smear out the inner vertices with $g \in \mathcal{S}\left(\mathbf{R}^{4}\right)$

$$
\int d^{4} x_{r+1} \ldots d^{4} x_{n} t\left(x_{1}-x_{n}, \ldots, x_{n-1}-x_{n}\right) g\left(x_{r+1}\right) \ldots g\left(x_{n}\right) \in \mathcal{S}^{\prime}\left(\mathbf{R}^{4 r}\right) .
$$

Next we consider the adiabatic limit $g \rightarrow 1$ of (1.26) in the sense of tempered distributions in $x_{1}, \ldots, x_{r}$. For this purpose we replace $g(x)$ by

$$
g_{\epsilon}(x) \stackrel{\text { def }}{=} g_{0}(\epsilon x), \quad g_{0}(0)=1,
$$

$g_{0} \in \mathcal{S}\left(\mathbf{R}^{4}\right)$ fixed, and consider $\epsilon \rightarrow 0, \epsilon>0$. Note

$$
\hat{g}_{\epsilon}(k)=\epsilon^{-4} \hat{g}_{0}\left(\frac{k}{\epsilon}\right) .
$$

Moreover we perform the Fourier transformation in the relative coordinates $y_{1} \stackrel{\text { def }}{=} x_{1}-$ $x_{r}, \ldots, y_{r-1} \stackrel{\text { def }}{=} x_{r-1}-x_{r}$

$$
\begin{gathered}
\frac{1}{(2 \pi)^{2(r-1)}} \int d^{4} y_{1} \ldots d^{4} y_{r-1} e^{i\left(p_{1} y_{1}+\ldots+p_{r-1} y_{r-1}\right)} \int d^{4} x_{r+1} \ldots d^{4} x_{n} \\
t\left(x_{1}-x_{n}, \ldots, x_{n-1}-x_{n}\right) g_{\epsilon}\left(x_{r+1}\right) \ldots g_{\epsilon}\left(x_{n}\right)= \\
=\int d^{4} k_{r+1} \ldots d^{4} k_{n} \hat{g}_{0}\left(k_{r+1}\right) \ldots \hat{g}_{0}\left(k_{n}\right) e^{-i \epsilon\left(k_{r+1}+\ldots+k_{n}\right) x_{r}} \\
\hat{t}\left(p_{1}, \ldots, p_{r-1},-\left(p_{1}+\ldots+p_{r-1}\right)+\epsilon\left(k_{r+1}+\ldots+k_{n}\right),-\epsilon k_{r+1}, \ldots,-\epsilon k_{n-1}\right),
\end{gathered}
$$

and obtain a tempered distribution in $p_{1}, \ldots, p_{r-1}, x_{r}$. The precise formulation of our assumtion (1.24-25) reads: Every point $p \stackrel{\text { def }}{=}\left(p_{1}, \ldots, p_{r-1}\right)$ with all $p_{1}, \ldots, p_{r-1}$ and with $\tilde{p}_{r} \stackrel{\text { def }}{=}-\left(p_{1}+\ldots+p_{r-1}\right)$ off-shell has a neighbourhood $U_{p}$ such that in (1.29) the adiabatic limit $\epsilon \rightarrow 0$ exists on the space of test functions with support in $\left(p, x_{r}\right) \in U_{p} \times \mathbf{R}^{4}$.

(3) Our assumption (1.24-25) implies that the divergences with respect to inner vertices vanish in the infrared limit (1.24)

$$
\lim _{p_{r+1}, \ldots, p_{n-1}, p_{n} \rightarrow 0} p_{l \alpha} \hat{t}_{\ldots}^{\alpha l \ldots}\left(p_{1}, \ldots, p_{n-1}\right)=0, \quad \forall l=r+1, \ldots, n .
$$

We shall see in sect.2 that $p_{l \alpha} \hat{t}_{\ldots}^{\alpha l \ldots}\left(p_{1}, \ldots, p_{n-1}\right), l=r+1, \ldots, n$, which contains one Q-vertex, can only be eliminated from the Cg-identities by taking the limit (1.30).

(4) To prove the Cg-identities in [4] it was necessary to assume (1.30) and that the infrared limit (1.24-25) of certain (not all) $\hat{t}$-distributions rsp. combinations of $\hat{t}$-distributions exists. However, we approached this limit there from totally space-like points only, where the $\hat{t}$-distributions are analytic. Especially we merely considered the region $p_{1}^{2}, \ldots, p_{r-1}^{2}, \tilde{p}_{r}^{2} \leq$ $-\delta<0$. This was sufficient, since we had to investigate a polynomial only, namely the possible violation of a certain Cg-identity. We see that our assumption (1.24-25) is quite stronger than the one [4] needed to prove the Cg-identities. 
(5) In sect.3 we consider connected $r$-point Green's functions with the external momenta $k_{1}, k_{2}, \ldots, k_{r}$, where $k_{1}+k_{2}+\ldots+k_{r}=0$. Their perturbative expansions will be written in terms of our $\hat{t}$-distributions by means of the Gell-Mann Low series. In doing so, all inner and the sum of the external momenta of the $\hat{t}$-distributions are set equal to zero. Considering the region with all $k_{I} \stackrel{\text { def }}{=} \sum_{i \in I} k_{i}$ off-shell (where $I$ runs through all subsets of $\{1,2, . ., r\}$ with $1,2, \ldots, r-1$ elements), the individual terms in these series exist if and only if our assumption (1.24-25) holds true for the corresponding $\hat{t}$-distributions. This will be evident by considering the explicit formulas. Usually the existence of the Gell-Mann Low expressions in the mentioned region is implicitly assumed in the literature. A perturbative formulation of the Slavnov-Taylor identities is impossible without our assumption (1.24-25).

\section{Elimination of the Distributions with one Q-Vertex in the Cg-Identities}

\section{(a) Two-Legs Cg-Identities}

The 2-legs Cg-identities are derived and proven in sect.3 of [2]. Since numerical distributions with an external $A$ - or $F$-line at the same vertex get combined in Green's functions or matrix elements, we define

$$
\begin{gathered}
\tilde{t}_{A A}^{\alpha \nu}\left(p_{1}, p_{2}, \ldots p_{n-1}\right) \stackrel{\text { def }}{=} t_{A A}^{\alpha \nu}\left(p_{1}, p_{2}, \ldots\right)+2 i p_{1 \tau} t_{F A}^{\tau \alpha \nu}\left(p_{1}, p_{2}, \ldots\right)+2 i p_{2 \tau} t_{A F}^{\alpha \tau \nu}\left(p_{1}, p_{2}, \ldots\right)- \\
-4 p_{1 \tau} p_{2 \rho} t_{F F}^{\tau \alpha \rho \nu}\left(p_{1}, p_{2}, \ldots\right)
\end{gathered}
$$

and

$$
\tilde{t}_{u A}^{\alpha l \nu}\left(p_{1}, p_{2}, \ldots p_{n-1}\right) \stackrel{\text { def }}{=} t_{u A}^{\alpha l \nu}\left(p_{1}, p_{2}, \ldots\right)+2 i p_{2 \tau} t_{u F}^{\alpha l \tau \nu}\left(p_{1}, p_{2}, \ldots\right) .
$$

In order to eliminate the distributions with one Q-vertex, we insert the 2-legs Cg-identities into each other and obtain

$$
0=p_{1 \alpha} \tilde{t}_{A A}^{\alpha \nu}\left(p_{1}, p_{2}, \ldots\right)-i\left[p_{2}^{2} t_{u \tilde{u}}^{\nu}\left(p_{1}, p_{2}, \ldots\right)-p_{2 \mu} p_{2}^{\nu} t_{u \tilde{u}}^{\mu}\left(p_{1}, p_{2}, \ldots\right)\right]+\sum_{l=3}^{n} p_{l \alpha} \tilde{t}_{u A}^{\alpha l \nu}\left(p_{1}, p_{2}, \ldots\right)
$$

where $p_{n} \stackrel{\text { def }}{=}-\left(p_{1}+p_{2}+\ldots+p_{n-1}\right)$. We recognize that the $\tilde{t}$-distributions $(2.1),(2.2)$ are well suited for this combined Cg-identity (2.3). The latter equation still contains distributions with one Q-vertex, namely in the divergences with respect to inner vertices $\sum_{l=3}^{n} p_{l \alpha} \tilde{t}_{u A}^{\alpha l \nu}\left(p_{1}, p_{2}, \ldots\right)$. In order to get rid of them we consider the infrared limit (1.24): $p_{3} \rightarrow 0, p_{4} \rightarrow 0, \ldots p_{n} \rightarrow 0$, with $p_{1}$ off-shell, and use (1.30). Taking additionally the covariant decomposition

$$
t_{u \tilde{u}}^{\mu}(p,-p, 0 \ldots)=p^{\mu} \bar{t}_{u \tilde{u}}\left(p^{2}\right)
$$

into account, we obtain for (2.3) the following simple reduced Cg-identity

$$
0=p_{\alpha} \tilde{t}_{A A}^{\alpha \nu}(p,-p, 0 \ldots)
$$




\section{(b) Three-Legs Cg-Identities}

We proceed in the same way for the 3-legs identities. First we consider the case with a pair $(\bar{\psi}, \psi)$ among the external legs. The corresponding Cg-identities are given and proven in sect.4 of [5]. Defining analogously to (2.1)

$$
\tilde{\tau}_{\bar{\psi} \psi A}^{\nu}\left(p_{1}, p_{2}, p_{3}, p_{4} \ldots, p_{n-1}\right) \stackrel{\text { def }}{=} \tau_{\bar{\psi} \psi A}^{\nu}\left(p_{1}, p_{2}, p_{3}, p_{4} \ldots\right)+2 i p_{3 \alpha} \tau \frac{\alpha \nu}{\psi} \psi_{\psi F}\left(p_{1}, p_{2}, p_{3}, p_{4} \ldots\right),
$$

we get by inserting (4.27) (of [5]) into (4.32) (of [5])

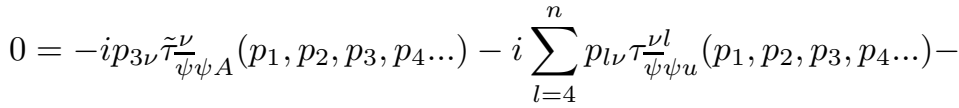

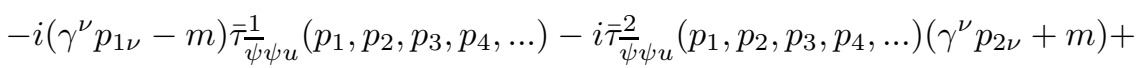

$$
\begin{aligned}
& +\frac{g}{2(2 \pi)^{2}}\left[i \tau_{\bar{\psi} \psi}\left(p_{1}+p_{3}, p_{2}, p_{4}, \ldots\right)-i \tau_{\bar{\psi} \psi}\left(p_{1}, p_{2}+p_{3}, p_{4}, \ldots\right)+\gamma_{\mu} t_{u \tilde{u}}^{\mu}\left(p_{3}, p_{1}+p_{2}, p_{4}, \ldots\right)\right],
\end{aligned}
$$

where again $p_{n} \stackrel{\text { def }}{=}-\left(p_{1}+p_{2}+\ldots+p_{n-1}\right)$. The divergences with respect to inner vertices, each containing one Q-vertex, can be eliminated by taking the infrared limit

$$
p_{4} \rightarrow 0, \ldots p_{n-1} \rightarrow 0, p_{n} \rightarrow 0, \quad p_{1}, p_{2}, p_{3} \text { off }- \text { shell },
$$

and using (1.30)

$$
\begin{gathered}
0=-i p_{3 \nu} \tilde{\tau}_{\bar{\psi} \psi A}^{\nu}\left(p_{1}, p_{2}, p_{3}, 0 \ldots\right)-i\left(\gamma^{\nu} p_{1 \nu}-m\right) \bar{\tau} \bar{\psi}_{\psi u}\left(p_{1}, p_{2}, p_{3}, 0, \ldots\right)- \\
-i \bar{\tau}_{\bar{\psi} \psi u}^{2}\left(p_{1}, p_{2}, p_{3}, 0, \ldots\right)\left(\gamma^{\nu} p_{2 \nu}+m\right)+\frac{g}{2(2 \pi)^{2}}\left[i \tau_{\bar{\psi} \psi}\left(p_{1}+p_{3}, p_{2}, 0, \ldots\right)-\right. \\
\left.-i \tau_{\bar{\psi} \psi}\left(p_{1}, p_{2}+p_{3}, 0, \ldots\right)+\gamma_{\mu} t_{u \tilde{u}}^{\mu}\left(p_{3}, p_{1}+p_{2}, 0, \ldots\right)\right]\left.\right|_{p_{1}+p_{2}+p_{3}=0 .}
\end{gathered}
$$

This reduced Cg-identity still contains distributions with one Q-vertex: $\bar{\tau} \frac{1}{\psi} \psi u, \bar{\tau}_{\bar{\psi} \psi u}^{2}$. It is not possible to eliminate them, except the external momenta $p_{1}, p_{2}$ approach the mass-shell: $\gamma^{\nu} p_{1 \nu} \rightarrow m, \gamma^{\nu} p_{2 \nu} \rightarrow-m$. But in the latter limit infrared divergences appear. Therefore, we avoid it.

We turn to the Cg-identities without external matter lines, derived in [3] and proven in [4]. We define

$$
\begin{gathered}
\tilde{t}_{A u \tilde{u}}^{\alpha \mu}\left(p_{1}, p_{2}, p_{3}, p_{4} \ldots, p_{n-1}\right) \stackrel{\text { def }}{=} t_{A u \tilde{u}}^{\alpha \mu}\left(p_{1}, p_{2}, p_{3}, p_{4} \ldots\right)+2 i p_{1 \beta} t_{F u \tilde{u}}^{\beta \alpha \mu}\left(p_{1}, p_{2}, p_{3}, p_{4} \ldots\right) \\
\tilde{t}_{A A A}^{\alpha \mu \nu}\left(p_{1}, p_{2}, p_{3}, p_{4} \ldots, p_{n-1}\right) \stackrel{\text { def }}{=} t_{A A A}^{\alpha \mu \nu}\left(p_{1}, p_{2}, p_{3}, \ldots\right)+2 i p_{1 \rho} t_{F A A}^{\rho \alpha \mu \nu}\left(p_{1}, p_{2}, p_{3}, \ldots\right)+ \\
+2 i p_{2 \rho} t_{A F A}^{\alpha \rho \mu \nu}\left(p_{1}, p_{2}, p_{3}, \ldots\right)+2 i p_{3 \rho} t_{A A F}^{\alpha \mu \rho \nu}\left(p_{1}, p_{2}, p_{3}, \ldots\right)-4 p_{1 \rho} p_{2 \tau} t_{F F A}^{\rho \alpha \mu \nu \nu}\left(p_{1}, p_{2}, p_{3}, \ldots\right)- \\
-4 p_{1 \rho} p_{3 \tau} t_{F A F}^{\rho \alpha \mu \tau \nu}\left(p_{1}, p_{2}, p_{3}, \ldots\right)-4 p_{2 \rho} p_{3 \tau} t_{A F F}^{\alpha \rho \tau \nu}\left(p_{1}, p_{2}, p_{3}, \ldots\right)- \\
-8 i p_{1 \rho} p_{2 \tau} p_{3 \lambda} t_{F F F}^{\rho \alpha \tau \mu \lambda \nu}\left(p_{1}, p_{2}, p_{3}, \ldots\right)- \\
-\frac{2 g}{(2 \pi)^{2}}\left[\left(t_{A F}^{\alpha \nu \mu}\left(p_{1}, p_{2}+p_{3}, p_{4}, \ldots, p_{n-1}\right)+2 i p_{1 \tau} t_{F F}^{\tau \alpha \nu \mu}\left(p_{1}, p_{2}+p_{3}, p_{4}, \ldots\right)\right)+\right.
\end{gathered}
$$




$$
\begin{aligned}
& +\left(t_{A F}^{\mu \alpha \nu}\left(p_{2}, p_{1}+p_{3}, p_{4}, \ldots\right)+2 i p_{2 \tau} t_{F F}^{\tau \mu \alpha \nu}\left(p_{2}, p_{1}+p_{3}, p_{4}, \ldots\right)\right)+ \\
& \left.+\left(t_{A F}^{\nu \mu \alpha}\left(p_{3}, p_{1}+p_{2}, p_{4}, \ldots\right)+2 i p_{3 \tau} t_{F F}^{\tau \nu \mu \alpha}\left(p_{3}, p_{1}+p_{2}, p_{4}, \ldots\right)\right)\right]
\end{aligned}
$$

and

$$
\begin{gathered}
\tilde{t}_{u A A}^{\alpha l \mu \nu}\left(p_{1}, p_{2}, p_{3}, p_{4} \ldots, p_{n-1}\right) \stackrel{\text { def }}{=} t_{u A A}^{\alpha l \mu \nu}\left(p_{1}, p_{2}, p_{3}, p_{4} \ldots\right)+2 i p_{2 \rho} t_{u F A}^{\alpha l \rho \mu \nu}\left(p_{1}, p_{2}, p_{3}, p_{4}, \ldots\right)+ \\
+2 i p_{3 \rho} t_{u A F}^{\alpha l \mu \rho \nu}\left(p_{1}, p_{2}, p_{3}, p_{4} \ldots\right)-4 p_{2 \rho} p_{3 \tau} t_{u F F}^{\alpha l \rho \mu \tau \nu}\left(p_{1}, p_{2}, p_{3}, p_{4} \ldots\right)+ \\
+\frac{2 g}{(2 \pi)^{2}} t_{u F}^{\alpha(l-1) \mu \nu}\left(p_{1}, p_{2}+p_{3}, p_{4}, \ldots\right)
\end{gathered}
$$

Note that the 2-legs distributions in (2.11), (2.12) are of order $(n-1)$. (2.10) and the 3-legs terms in (2.11), (2.12) are motivated by the combination of external $A$ - and $F$-lines at the same vertex, similarly to (2.1), (2.2) and (2.6). The 2-legs terms in (2.11), (2.12) are terms with an external four-gluon vertex (1.18). The diagram for the term $\sim g t_{A F}^{\alpha \nu \mu}\left(p_{1}, p_{2}+\right.$ $\left.p_{3}, p_{4}, \ldots, . p_{n-1}\right)$ is given in fig. 1 in $x$-space: The four-gluon vertex at $x_{2}=x_{3}$ on the l.h.s. is generated by the normalization term $-\frac{1}{2} \delta\left(x_{2}-x_{3}\right)$ of the propagator $\partial \partial D_{F}\left(x_{2}-x_{3}\right)$ on the r.h.s.. Now we eliminate the distributions with one Q-vertex in the list of 3-legs Cg-identities given in sect.3 of [3]. For this purpose the identities of type I have already been inserted into the identities of type II in this list. We eliminate $t_{u A F}^{2}$ by inserting (3.14) into (3.13) (antisymmetrized in $\left.\left(x_{2} / p_{2}, \mu\right) \leftrightarrow\left(x_{3} / p_{3}, \nu\right)\right)$. Inserting then the resulting equation into (3.12) we get rid of $t_{u A A}^{3}$. Moreover, $t_{u A}^{2}$ in (3.12b) is eliminated by applying the 2-legs Cg-identity (3.20) of [2]. We finally obtain

$$
\begin{gathered}
0=-i p_{1 \alpha} \tilde{t}_{A A A}^{\alpha \mu \nu}\left(p_{1}, p_{2}, p_{3}, p_{4} \ldots\right)-i \sum_{l=4}^{n} p_{l \alpha} \tilde{t}_{u A A}^{\alpha l \mu \nu}\left(p_{1}, p_{2}, p_{3}, p_{4} \ldots\right)+p_{3}^{2} \tilde{t}_{A u \tilde{u}}^{\mu \nu}\left(p_{2}, p_{1}, p_{3}, p_{4}, \ldots\right)- \\
\quad-p_{3}^{\nu} p_{3 \alpha} \tilde{t}_{A u \tilde{u}}^{\mu \alpha}\left(p_{2}, p_{1}, p_{3}, p_{4}, \ldots\right)-p_{2}^{2} \tilde{t}_{A u \tilde{u}}^{\nu \mu}\left(p_{3}, p_{1}, p_{2}, p_{4}, \ldots\right)+p_{2}^{\mu} p_{2 \alpha} \tilde{t}_{A u \tilde{u}}^{\nu \alpha}\left(p_{3}, p_{1}, p_{2}, p_{4}, \ldots\right)+ \\
+\frac{g}{(2 \pi)^{2}}\left[\tilde{t}_{A A}^{\mu \nu}\left(p_{1}+p_{2}, p_{3}, p_{4}, \ldots\right)-\tilde{t}_{A A}^{\nu \mu}\left(p_{1}+p_{3}, p_{2}, p_{4}, \ldots\right)+i\left[\left(2 p_{3}^{\mu}+p_{2}^{\mu}\right) t_{u \tilde{u}}^{\nu}\left(p_{1}, p_{2}+p_{3}, p_{4}, \ldots\right)-\right.\right. \\
\left.\left.-g^{\mu \nu} p_{3 \alpha} t_{u \tilde{u}}^{\alpha}\left(p_{1}, p_{2}+p_{3}, p_{4}, \ldots\right)-\left(2 p_{2}^{\nu}+p_{3}^{\nu}\right) t_{u \tilde{u}}^{\mu}\left(p_{1}, p_{2}+p_{3}, p_{4}, \ldots\right)+g^{\mu \nu} p_{2 \alpha} t_{u \tilde{u}}^{\alpha}\left(p_{1}, p_{2}+p_{3}, p_{4} \ldots\right)\right]\right] .
\end{gathered}
$$

Without introducing the $\tilde{t}$-distributions, this combined Cg-identity would be much more complicated. This is another motivation for the above definitions. Again we take the limit (2.8) and apply (1.30). Using additionally (2.4) we get the reduced Cg-identity

$$
\begin{gathered}
0=-i p_{1 \alpha} \tilde{t}_{A A \mu}^{\alpha \mu \nu}\left(p_{1}, p_{2}, p_{3}, 0, \ldots\right)+p_{3}^{2} \tilde{t}_{A u \tilde{u}}^{\mu \nu}\left(p_{2}, p_{1}, p_{3}, 0, \ldots\right)-p_{3}^{\nu} p_{3 \alpha} \tilde{t}_{A u \tilde{u}}^{\mu \alpha}\left(p_{2}, p_{1}, p_{3}, 0, \ldots\right)- \\
-p_{2}^{2} \tilde{t}_{A u \tilde{u}}^{\nu \mu}\left(p_{3}, p_{1}, p_{2}, 0, \ldots\right)+p_{2}^{\mu} p_{2 \alpha} \tilde{t}_{A u \tilde{u}}^{\nu \alpha}\left(p_{3}, p_{1}, p_{2}, 0, \ldots\right)+ \\
\quad+\frac{g}{(2 \pi)^{2}}\left[\tilde{t}_{A A}^{\mu \nu}\left(-p_{3}, p_{3}, 0, \ldots\right)-\tilde{t}_{A A}^{\nu \mu}\left(-p_{2}, p_{2}, 0, \ldots\right)+\right. \\
\left.+i \bar{t}_{u \tilde{u}}\left(p_{1}^{2}\right)\left[p_{3}^{2} g^{\mu \nu}-p_{3}^{\mu} p_{3}^{\nu}-p_{2}^{2} g^{\mu \nu}+p_{2}^{\mu} p_{2}^{\nu}\right]\right]\left.\right|_{p_{1}+p_{2}+p_{3}=0 .}
\end{gathered}
$$

Only distributions of the physical theory (i.e. distributions without Q-vertex) appear in this equation.

There remains the 3-legs Cg-identity (3.9) of [3]. Going again over to the infrared limit (2.8), this Cg-identity is reduced by means of (1.30) to

$$
0=-i p_{1 \alpha} \tilde{t}_{A u \tilde{u}}^{\alpha \mu}\left(p_{1}, p_{2}, p_{3}, 0 \ldots\right)-i p_{2 \alpha} \tilde{t}_{A u \tilde{u}}^{\alpha \mu}\left(p_{2}, p_{1}, p_{3}, 0 \ldots\right)-i p_{3}^{\mu} \vec{t}_{u u \tilde{u}}^{3}\left(p_{1}, p_{2}, p_{3}, 0 \ldots\right)+
$$




$$
+\left.\frac{g}{(2 \pi)^{2}}\left[t_{u \tilde{u}}^{\mu}\left(p_{1}+p_{2}, p_{3}, 0 \ldots\right)-t_{u \tilde{u}}^{\mu}\left(p_{2}, p_{1}+p_{3}, 0 \ldots\right)-t_{u \tilde{u}}^{\mu}\left(p_{1}, p_{2}+p_{3}, 0 \ldots\right)\right]\right|_{p_{1}+p_{2}+p_{3}=0 .} .
$$

However, $\bar{t}_{u u \tilde{u}}^{3}$, which contains one Q-vertex, cannot be eliminated.

\section{(c) Four-Legs Cg-Identities}

To shorten the story we immediately take the limit

$$
p_{5}, \ldots p_{n-1}, p_{n} \rightarrow 0, \quad p_{k},\left(p_{i}+p_{j}\right) \text { off }- \text { shell } \forall k=1, \ldots, 4, \forall 1 \leq i<j \leq 4 .
$$

Besides $p_{k}$, the momenta $\left(p_{i}+p_{j}\right)$ need to be off-shell too, since they appear in the arguments of the 3- or 2-legs distributions in the degenerate terms. (The latter include terms with external four-gluon vertex/vertices.) Due to (1.30), the divergences with respect to inner vertices vanish in this limit (2.16). We define

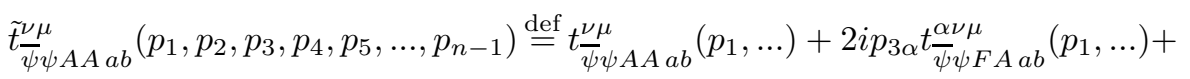

$$
\begin{aligned}
& +2 i p_{4 \alpha} t \frac{\nu \alpha \mu}{\psi} \psi_{\psi A F a b}\left(p_{1}, \ldots\right)-4 p_{3 \alpha} p_{4 \beta} t_{\bar{\psi} \psi F F a b}^{\alpha \nu \beta \mu}\left(p_{1}, \ldots\right)+\frac{2 g}{(2 \pi)^{2}} \tau \frac{\nu \mu}{\psi} \psi_{F}\left(p_{1}, p_{2}, p_{3}+p_{4}, p_{5}, \ldots\right) f_{a b c} \lambda_{c}, \\
& \tilde{t}_{\bar{\psi} \psi u A}^{1 \mu}\left(p_{1}, p_{2}, p_{3}, p_{4}, p_{5}, \ldots, p_{n-1}\right) \stackrel{\text { def }}{=} \bar{t}_{\bar{\psi} \psi u A}^{1 \mu}\left(p_{1}, \ldots\right)+2 i p_{4 \alpha} \bar{t}_{\bar{\psi} \psi u F}^{1 \alpha \mu}\left(p_{1}, \ldots\right) \\
& \tilde{t}_{\bar{\psi} \psi u A}^{2 \mu}\left(p_{1}, p_{2}, p_{3}, p_{4}, p_{5}, \ldots, p_{n-1}\right) \stackrel{\text { def }}{=} \bar{t}_{\bar{\psi} \psi u A}^{2 \mu}\left(p_{1}, \ldots\right)+2 i p_{4 \alpha} \vec{t}_{\bar{\psi} \psi u F}^{2 \alpha \mu}\left(p_{1}, \ldots\right), \\
& \tilde{t}_{u \tilde{u} A A a b c d}^{\tau \nu \mu}\left(p_{1}, p_{2}, p_{3}, p_{4}, p_{5}, \ldots, p_{n-1}\right) \stackrel{\text { def }}{=} t_{u \tilde{u} A A a b c d}^{\tau \nu \mu}\left(p_{1}, \ldots\right)+2 i p_{3 \alpha} t_{u \tilde{u} F A a b c d}^{\tau \alpha \mu \mu}\left(p_{1}, \ldots\right)+ \\
& +2 i p_{4 \alpha} t_{u \tilde{u} A F a b c d}^{\tau \nu \alpha \mu}\left(p_{1}, \ldots\right)-4 p_{3 \alpha} p_{4 \beta} t_{u \tilde{u} F F a b c d}^{\tau \alpha \nu \beta \mu}\left(p_{1}, \ldots\right)+\frac{2 g}{(2 \pi)^{2}} t_{u \tilde{u} F}^{\tau \nu \mu}\left(p_{1}, p_{2}, p_{3}+p_{4}, p_{5}, \ldots\right) f_{a b r} f_{c d r}, \\
& \tilde{t}_{A A A A a b c d}^{\alpha \nu \kappa \lambda}\left(p_{1}, p_{2}, p_{3}, p_{4}, p_{5}, \ldots, p_{n-1}\right) \stackrel{\text { def }}{=} t_{A A A A \lambda a b c d}^{\alpha \nu \kappa \lambda}\left(p_{1}, \ldots\right)+2 i p_{1 \beta} t_{F A A A a b c d}^{\beta \alpha \nu \kappa \lambda}\left(p_{1}, \ldots\right)+ \\
& +2 i p_{2 \gamma} t_{A F A A \text { abcd }}^{\alpha \gamma \nu \kappa \lambda}\left(p_{1}, \ldots\right)+2 i p_{3 \rho} t_{A A F A a b c d}^{\alpha \nu \rho \kappa \lambda}\left(p_{1}, \ldots\right)+2 i p_{4 \tau} t_{A A A F a b c d}^{\alpha \nu \kappa \tau \lambda}\left(p_{1}, \ldots\right)- \\
& -4 p_{1 \beta} p_{2 \gamma} t_{F F A A \text { abcd }}^{\beta \alpha \gamma \kappa \lambda}\left(p_{1}, \ldots\right)-4 p_{1 \beta} p_{3 \rho} t_{F A F A \text { abcd }}^{\beta \alpha \nu \rho \kappa}\left(p_{1}, \ldots\right)-4 p_{1 \beta} p_{4 \tau} t_{F A A F \text { abcd }}^{\beta \alpha \mu \kappa \lambda}\left(p_{1}, \ldots\right)- \\
& -4 p_{2 \gamma} p_{3 \rho} t_{A F F A \text { abcd }}^{\alpha \gamma \nu \kappa \lambda}\left(p_{1}, \ldots\right)-4 p_{2 \gamma} p_{4 \tau} t_{A F A F \text { abcd }}^{\alpha \gamma \nu \kappa \tau \lambda}\left(p_{1}, \ldots\right)- \\
& -4 p_{3 \rho} p_{4 \tau} t_{A A F F \text { abcd }}^{\alpha \nu \rho \kappa \tau \lambda}\left(p_{1}, \ldots\right)-8 i p_{1 \beta} p_{2 \gamma} p_{3 \rho} t_{F F F A \text { abcd }}^{\beta \alpha \nu \rho \kappa \lambda}\left(p_{1}, \ldots\right)- \\
& -8 i p_{1 \beta} p_{2 \gamma} p_{4 \tau} t_{F F A F \text { abcd }}^{\beta \alpha \gamma \nu \kappa \tau \lambda}\left(p_{1}, \ldots\right)-8 i p_{1 \beta} p_{3 \rho} p_{4 \tau} t_{F A F F, a b c d}^{\beta \alpha \nu \rho \kappa \tau \lambda}\left(p_{1}, \ldots\right)- \\
& -8 i p_{2 \gamma} p_{3 \rho} p_{4 \tau} t_{A F F F \text { abcd }}^{\alpha \gamma \nu \rho \kappa \tau \lambda}\left(p_{1}, \ldots\right)+16 p_{1 \beta} p_{2 \gamma} p_{3 \rho} p_{4 \tau} t_{F F F F \text { abcd }}^{\beta \alpha \gamma \nu \kappa \tau \lambda}\left(p_{1}, \ldots\right)+ \\
& +\frac{2 g}{(2 \pi)^{2}}\left\{f _ { a b r } f _ { c d r } \left[t_{A A F}^{\alpha \nu \kappa \lambda}\left(p_{1}, p_{2}, p_{3}+p_{4}, p_{5}, \ldots\right)+2 i p_{1 \beta} t_{F A F}^{\beta \alpha \nu \kappa \lambda}\left(p_{1}, p_{2}, p_{3}+p_{4}, p_{5}, \ldots\right)+\right.\right. \\
& +2 i p_{2 \gamma} t_{A F F}^{\alpha \gamma \nu \kappa \lambda}\left(p_{1}, p_{2}, p_{3}+p_{4}, p_{5}, \ldots\right)-4 p_{1 \beta} p_{2 \gamma} t_{F F F}^{\beta \alpha \gamma \nu \kappa \lambda}\left(p_{1}, p_{2}, p_{3}+p_{4}, p_{5}, \ldots\right)+ \\
& +t_{A A F}^{\kappa \lambda \alpha \nu}\left(p_{3}, p_{4}, p_{1}+p_{2}, p_{5}, \ldots\right)+2 i p_{3 \rho} t_{F A F}^{\rho \kappa \lambda \alpha \nu}\left(p_{3}, p_{4}, p_{1}+p_{2}, p_{5}, \ldots\right)+ \\
& +2 i p_{4 \tau} t_{A F F}^{\kappa \tau \lambda \alpha \nu}\left(p_{3}, p_{4}, p_{1}+p_{2}, p_{5}, \ldots\right)-4 p_{3 \rho} p_{4 \tau} t_{F F F}^{\rho \kappa \tau \lambda \alpha \nu}\left(p_{3}, p_{4}, p_{1}+p_{2}, p_{5}, \ldots\right)+
\end{aligned}
$$




$$
\begin{gathered}
\left.\left.+\frac{2 g}{(2 \pi)^{2}} t_{F F}^{\alpha \nu \kappa \lambda}\left(p_{1}+p_{2}, p_{3}+p_{4}, p_{5}, \ldots\right)\right]\right\}+ \\
+\frac{2 g}{(2 \pi)^{2}}\left\{\text { one cyclic permutation }\left(b, p_{2}, \nu\right) \rightarrow\left(c, p_{3}, \kappa\right) \rightarrow\left(d, p_{4}, \lambda\right) \rightarrow\right. \\
\left.\rightarrow\left(b, p_{2}, \nu\right)\right\}+\frac{2 g}{(2 \pi)^{2}}\{\text { two cyclic permutations }\}
\end{gathered}
$$

and

$$
\tilde{t}_{u u \tilde{u} A}^{3 \nu}\left(p_{1}, \ldots, p_{n-1}\right) \stackrel{\text { def }}{=} \bar{t}_{u u \tilde{u} A}^{3 \nu}\left(p_{1}, \ldots, p_{n-1}\right)+2 i p_{4 \tau} \bar{t}_{u u \tilde{u} F}^{3 \tau \nu}\left(p_{1}, \ldots, p_{n-1}\right) .
$$

The motivation for these definitions is the same as above. The terms with a 3-legs distribution are terms with one external four-gluon vertex, analogously to fig.1. The $t_{F F}$-terms in $\tilde{t}_{A A A A}$ have two external four-gluon vertices: The diagram in $\mathrm{x}$-spaxe belonging to the term $\sim g^{2} t_{F F}\left(p_{1}+p_{2}, p_{3}+p_{4}, p_{5}, \ldots\right)$ has one four-gluon vertex at $x_{1}=x_{2}$ and another at $x_{3}=x_{4}$.

First we consider the 4-legs Cg-identities with one pair $(\bar{\psi}, \psi)$ among the external legs. They are derived and proven in sect.4 of [5]. Inserting the identities of type I into the identities of type II and (4.34) (of [5]) into (4.33) (of [5]) we obtain the reduced Cg-identity

$$
\begin{gathered}
0=-i p_{3 \nu} \tilde{t}_{\bar{\psi} \psi A A a b}^{\nu \mu}\left(p_{1}, p_{2}, p_{3}, p_{4}, 0, \ldots\right)- \\
-i\left(\gamma^{\nu} p_{1 \nu}-m\right) \tilde{t}_{\frac{\psi}{\psi} \psi u A a b}^{\mu}\left(p_{1}, p_{2}, p_{3}, p_{4}, 0, \ldots\right)-i \tilde{t}_{\bar{\psi} \psi u A a b}^{\mu}\left(p_{1}, p_{2}, p_{3}, p_{4}, 0, \ldots\right)\left(\gamma^{\nu} p_{2 \nu}+m\right)+ \\
+p_{4}^{\mu} p_{4 \nu} t_{\bar{\psi} \psi u \tilde{u} a b}^{\nu}\left(p_{1}, p_{2}, p_{3}, p_{4}, 0, \ldots\right)-p_{4}^{2} t_{\bar{\psi} \psi u \tilde{u} a b}^{\mu}\left(p_{1}, p_{2}, p_{3}, p_{4}, 0, \ldots\right)+ \\
+\frac{g}{(2 \pi)^{2}}\left\{\frac{i}{2}\left[\lambda_{a} \lambda_{b} \tilde{\tau}_{\bar{\psi} \psi A}^{\mu}\left(p_{1}+p_{3}, p_{2}, p_{4}, 0, \ldots\right)-\lambda_{b} \lambda_{a} \tilde{\tau}_{\bar{\psi} \psi A}^{\mu}\left(p_{1}, p_{2}+p_{3}, p_{4}, 0, \ldots\right)\right]-\right. \\
-\frac{1}{2} \gamma_{\lambda} f_{a b c} \lambda_{c} \tilde{t}_{A u \tilde{u}}^{\mu \lambda}\left(p_{4}, p_{3}, p_{1}+p_{2}, 0, \ldots\right)+f_{a b c} \lambda_{c} \tilde{\tau}_{\bar{\psi} \psi A}^{\mu}\left(p_{1}, p_{2}, p_{3}+p_{4}, 0, \ldots\right)- \\
\left.-\frac{i}{2}\left[\gamma^{\mu} \lambda_{b} \lambda_{a} \bar{\tau} \frac{1}{\psi} \psi\left(p_{1}+p_{4}, p_{2}, p_{3}, 0, \ldots\right)-\bar{\tau}_{\bar{\psi} \psi u}^{2}\left(p_{1}, p_{2}+p_{4}, p_{3}, 0, \ldots\right) \lambda_{a} \lambda_{b} \gamma^{\mu}\right]\right\}\left.\right|_{p_{1}+p_{2}+p_{3}+p_{4}=0}
\end{gathered}
$$

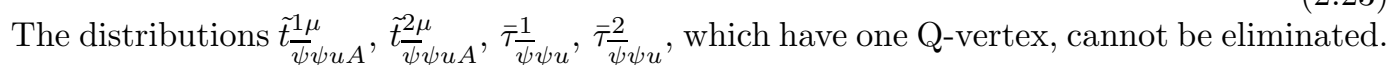

The 4-legs Cg-identities without external $\bar{\psi}, \psi$ are given in sect.4 of [3] and proven in [4]. We eliminate $t_{u A F F}^{2}$ by inserting (4.28) (of [3]) into (4.27). Then we insert the equation obtained in this way into (4.24), to get rid of $t_{u A A F}^{3}$. By substituting the resulting equation into (4.17), we eliminate $t_{u A A A}^{2}$. The 3-legs terms still contain distributions with one Qvertex. By using (3.13) of [3] we eliminate $t_{u A A}^{2}$ and then we get rid of $t_{u A F}^{2}$ by means of (3.14) (of [3]). The 2-legs terms $\sim t_{A F}$ and $\sim t_{F F}$ cancel due to Jacobi's identity. The result of this lengthy insertions is a reduced Cg-identity without any distribution with Q-vertex

$$
\begin{gathered}
0=-i p_{1 \alpha} \tilde{t}_{A A A A \mu a b c d}^{\alpha \nu \kappa \lambda}\left(p_{1}, p_{2}, p_{3}, p_{4}, 0, \ldots\right)+ \\
+\left[p_{2 \alpha} p_{2}^{\nu} \tilde{t}_{u \tilde{u} A A a b c d}^{\alpha \kappa \lambda}\left(p_{1}, p_{2}, p_{3}, p_{4}, 0, \ldots\right)-p_{2}^{2} \tilde{t}_{u \tilde{u} A A a b c d}^{\nu \kappa \lambda}\left(p_{1}, p_{2}, p_{3}, p_{4}, 0, \ldots\right)+\right. \\
+\frac{g}{(2 \pi)^{2}} f_{a b r} f_{c d r}\left\{\tilde{t}_{A A A}^{\nu \kappa \lambda}\left(p_{1}+p_{2}, p_{3}, p_{4}, 0, \ldots\right)+i\left(2 p_{3}+p_{4}\right)^{\lambda} \tilde{t}_{A u \tilde{u}}^{\nu \kappa}\left(p_{2}, p_{1}, p_{3}+p_{4}, 0, \ldots\right)-\right. \\
-i\left(2 p_{4}+p_{3}\right)^{\kappa} \tilde{t}_{A u \tilde{u}}^{\nu \lambda}\left(p_{2}, p_{1}, p_{3}+p_{4}, 0, \ldots\right)+i g^{\lambda \kappa}\left(p_{4}-p_{3}\right)_{\alpha} \tilde{t}_{A u \tilde{u}}^{\nu \alpha}\left(p_{2}, p_{1}, p_{3}+p_{4}, 0, \ldots\right)+
\end{gathered}
$$




$$
\begin{gathered}
\left.\left.+\frac{g}{(2 \pi)^{2}}\left[g^{\nu \kappa} t_{u \tilde{u}}^{\lambda}\left(p_{1},-p_{1}, 0, \ldots\right)-(\kappa \leftrightarrow \lambda)\right]\right\}\right]+ \\
+\left[\text { one cyclic permutation }\left(b, p_{2}, \nu\right) \rightarrow\left(c, p_{3}, \kappa\right) \rightarrow\left(d, p_{4}, \lambda\right) \rightarrow\right. \\
\left.\rightarrow\left(b, p_{2}, \nu\right)\right]+\left.[\text { two cyclic permutations }]\right|_{p_{1}+p_{2}+p_{3}+p_{4}=0 .}
\end{gathered}
$$

There remain the non-trivial 4-legs Cg-identities (4.20) and (4.29) in sect.4 of [3]. By inserting the latter into the first one, we eliminate $t_{u u \tilde{u} A}^{4}$. Moreover we eliminate $\bar{t}_{u u \tilde{u}}^{3}$ by means of $(2.15)$

$$
\begin{gathered}
0=-i\left[p_{1 \alpha} \tilde{t}_{u \tilde{u} A A b c a d}^{\mu \alpha \nu}\left(p_{2}, p_{3}, p_{1}, p_{4}, 0, \ldots\right)\right]+i\left[\left(a, p_{1}\right) \leftrightarrow\left(b, p_{2}\right)\right]- \\
-i p_{3}^{\mu} \tilde{t}_{u u \tilde{u} A a b c d}^{3 \nu}\left(p_{1}, p_{2}, p_{3}, p_{4}, 0, \ldots\right)+ \\
+p_{4}^{\nu} p_{4 \beta} t_{u u \tilde{u} \tilde{u} a b c d}^{\mu \beta}\left(p_{1}, p_{2}, p_{3}, p_{4}, 0, \ldots\right)-p_{4}^{2} t_{u u \tilde{u} \tilde{u} a b c d}^{\mu \nu}\left(p_{1}, p_{2}, p_{3}, p_{4}, 0, \ldots\right)+ \\
+\frac{g}{(2 \pi)^{2}}\left\{f _ { a b r } f _ { c d r } \left[\tilde{t}_{A u \tilde{u}}^{\nu \mu}\left(p_{4}, p_{1}+p_{2}, p_{3}, 0, \ldots\right)+\right.\right. \\
+\frac{g^{\nu \mu}}{\left(p_{1}+p_{2}\right)^{2}}\left\{p_{1 \alpha}\left(p_{1}+p_{2}\right)_{\beta} \tilde{t}_{A u \tilde{u}}^{\alpha \beta}\left(p_{1}, p_{2}, p_{3}+p_{4}, 0, \ldots\right)+p_{2 \alpha}\left(p_{1}+p_{2}\right)_{\beta} \tilde{t}_{A u \tilde{u}}^{\alpha \beta}\left(p_{2}, p_{1}, p_{3}+p_{4}, 0, \ldots\right)+\right. \\
\left.\left.+\frac{i g}{(2 \pi)^{2}}\left[\left(p_{1}+p_{2}\right)^{2} \bar{t}_{u \tilde{u}}\left(\left(p_{1}+p_{2}\right)^{2}\right)-\left(p_{1} p_{2}+p_{2}^{2}\right) \bar{t}_{u \tilde{u}}\left(p_{2}^{2}\right)-\left(p_{1} p_{2}+p_{1}^{2}\right) \bar{t}_{u \tilde{u}}\left(p_{1}^{2}\right)\right]\right\}\right]+ \\
+f_{a d r} f_{b c r}\left[\tilde{t}_{A u \tilde{u}}^{\nu \mu}\left(p_{1}+p_{4}, p_{2}, p_{3}, 0, \ldots\right)+\tilde{t}_{A u \tilde{u}}^{\nu \mu}\left(p_{4}, p_{1}, p_{2}+p_{3}, 0, \ldots\right)\right]+ \\
\left.\left.+f_{a c r} f_{d b r}\left[\tilde{t}_{A u \tilde{u}}^{\nu \mu}\left(p_{2}+p_{4}, p_{1}, p_{3}, 0, \ldots\right)+\tilde{t}_{A u \tilde{u}}^{\nu \mu}\left(p_{4}, p_{2}, p_{1}+p_{3}, 0, \ldots\right)\right]\right\}\left.\right|_{p_{1}+p_{2}+p_{3}+p_{4}=0 .} .25\right)
\end{gathered}
$$

This equation still contains a distribution with one Q-vertex, namely $\tilde{t}_{u u \tilde{u} A}^{3}$, which cannot be eliminated. Again, the introduction of the $\tilde{t}$-distributions simplifies the reduced Cg-identities (2.23-25) enormously.

\section{(d) Gauge Invariant (Finite) Renormalizations}

We study finite renormalizations in the above limits of vanishing inner momenta (including $\left.p_{n} \stackrel{\text { def }}{=}-\left(p_{1}+\ldots+p_{n-1}\right)\right)$ and with (sums of) the external momenta off-shell. Note that for the Gell-Mann Low series of the connected Green's functions (sect.3) we shall need the values of the $t$-distributions in these limits only. The freedom of normalization (1.16) of a numerical distribution $\hat{t}$ is a Lorentz covariant, $\mathrm{SU}(\mathrm{N})$-invariant polynomial (in $\left.p_{1}, p_{2}, \ldots, p_{n-1}\right)$ of degree $\omega$ which has the same permutation symmetries as $t[2,3,4,5]$, where $\omega$ is given by (1.17). Inserting these renormalization polynomials of the $t$ 's into the definition of $\tilde{t}$ (e.g. into (2.1)), we obtain the (undetermined) renormalization polynomial of $\tilde{t}$. These are the results:

$$
\begin{gathered}
\tilde{t}_{A A}^{\mu \nu}(p,-p, 0, \ldots)+C_{A A}^{1} g^{\mu \nu}+C_{A A}^{2} g^{\mu \nu} p^{2}+C_{A A}^{3} p^{\mu} p^{\nu} \\
t_{u \tilde{u}}^{\nu}(p,-p, 0, \ldots)+i C_{u \tilde{u}} p^{\nu} \\
\tau_{\bar{\psi} \psi}(p,-p, 0, \ldots)+C_{\bar{\psi} \psi}^{0}+C_{\bar{\psi} \psi} \gamma^{\nu} p_{\nu}
\end{gathered}
$$




$$
\begin{aligned}
& \tilde{t}_{A A A}^{\nu \kappa \lambda}\left(p_{1}, p_{2}, p_{3}, 0, \ldots\right)-\left.i C_{A A A}\left[g^{\nu \kappa}\left(p_{1}-p_{2}\right)^{\lambda}+g^{\nu \lambda}\left(p_{3}-p_{1}\right)^{\kappa}+g^{\kappa \lambda}\left(p_{2}-p_{3}\right)^{\nu}\right]\right|_{p_{1}+p_{2}+p_{3}=0}, \\
& \tilde{t}_{A u \tilde{u}}^{\mu \nu}+g^{\mu \nu} C_{A u \tilde{u}}, \\
& \tilde{\tau}_{\bar{\psi} \psi A}^{\nu}+C_{\bar{\psi} \psi A} \gamma^{\nu} \text {, }
\end{aligned}
$$

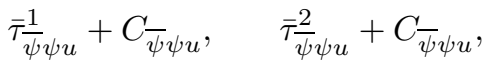

$$
\begin{aligned}
& \vec{t}_{u u \tilde{u}}^{3}+C_{u u \tilde{u}}, \\
& \tilde{t}_{A A A A a b c d}^{\alpha \nu \kappa \lambda}+C_{A A A A}\left\{\left[f_{a b r} f_{c d r}\left(-g^{\alpha \lambda} g^{\nu \kappa}+g^{\alpha \kappa} g^{\nu \lambda}\right)\right]+\right. \\
& +[\text { one cyclic permutation }(b, \nu) \rightarrow(c, \kappa) \rightarrow(d, \lambda) \rightarrow(b, \nu)]+ \\
& +[\text { two cyclic permutations }]\}+N_{A A A A a b c d}^{\alpha \nu \kappa \lambda} \text {. }
\end{aligned}
$$

The other 4-legs distributions cannot be renormalized, because their singular order is $\omega \leq$ -1 . The equality of the renormalization terms of $\bar{\tau} \frac{1}{\psi} \psi_{u}$ and $\bar{\tau} \frac{2}{\psi} \psi_{u}$ in (2.32) is a consequence of C-invariance [5]. The colour space of the 4-legs distributions was studied in [4] (sect.3(a) and appendix A). It is 5 - or 6 -dimensional. The linear span of the tensors $f_{. . r} f_{. . r}$ is a 2 dimensional subspace. $N_{A A A A a b c d}$ in (2.34) is an element of a linear complement of this subspace. For our purposes it is not necessary to determind the form of $N_{A A A A}$ abcd explicitly.

We assume that the initial $t, \tilde{t}$-distributions fulfil our reduced $\mathrm{Cg}$-identities and require that the renormalized ones fulfil them, too. This gives the following restrictions on the renormalization constants in (2.26-34): From (2.5)

$$
C_{A A}^{1}=0, \quad C_{A A}^{2}=-C_{A A}^{3}\left(\stackrel{\text { def }}{=} C_{A A}\right)
$$

from $(2.9)$

$$
0=-C_{\bar{\psi} \psi A}+C_{\bar{\psi} \psi u}+\frac{g}{2(2 \pi)^{2}}\left(C_{\bar{\psi} \psi}+C_{u \tilde{u}}\right)
$$

from $(2.14)$

$$
0=-C_{A A A}+C_{A u \tilde{u}}+\frac{g}{(2 \pi)^{2}}\left(C_{A A}-C_{u \tilde{u}}\right)
$$

from $(2.15)$

$$
C_{u u \tilde{u}}=C_{A u \tilde{u}}
$$

from (2.23)

$$
C_{\bar{\psi} \psi u}=-\frac{1}{2} C_{A u \tilde{u}}
$$

from $(2.24)$

$$
N_{A A A A a b c d}^{\alpha \nu \kappa \lambda}=0, \quad C_{A A A A}=\frac{g}{(2 \pi)^{2}}\left(C_{A A A}+C_{A u \tilde{u}}-\frac{g}{(2 \pi)^{2}} C_{u \tilde{u}}\right)
$$

and (2.25) implies no restriction, due to the Jacobi identity. Assuming $C_{A A A A} \sim g^{n}$, the 3 -legs (2-legs) normalization constants are of order $(n-1)$ (rsp. $(n-2))$.

Every reduced Cg-identity (2.9), (2.23), (2.15) and (2.25) still contains one Q-vertex. However, there is a big difference between the first two and the latter two: (2.15) and 
(2.25) yield (2.38) only, which is physically of no importance, since $\bar{t}_{u u \tilde{u}}^{3}(2.33)$ has one Qvertex. These two reduced Cg-identities are irrelevant for the normalization of the physical $t$-distributions. On the other hand, $C_{\bar{\psi} \psi u}$, which belongs to the unphysical distributions $\bar{\tau} \frac{1}{\psi} \psi u, \bar{\tau} \bar{\psi}_{\psi u}$ in (2.9), (2.23), can be eliminated by inserting (2.39) into (2.36). Therefore, (2.9) and (2.23) give restrictions on the physical theory. Apparantly this was the motivation for Taylor [10] to study the corresponding Slavnov-Taylor identities (sect.3), although he had to introduce the unphysical Q-vertex for this purpose. The result of the above mentioned insertion and (2.40) can be written in the form

$$
\begin{gathered}
0=-C_{\bar{\psi} \psi A}-\frac{1}{2} C_{A A A}+\frac{g}{2(2 \pi)^{2}}\left(C_{\bar{\psi} \psi}+C_{A A}\right), \\
C_{A A A A}=\frac{g}{(2 \pi)^{2}}\left(2 C_{A A A}-\frac{g}{(2 \pi)^{2}} C_{A A}\right),
\end{gathered}
$$

where (2.37) has been used.

Remarks: (A) The renormalizations (2.26-34) with the restrictions (2.35-42) are the most general finite renormalizations of the $\tilde{t}$-distributions (rsp. $t$-distributions in the cases where no $\tilde{t}$ was defined) which conserve the reduced Cg-identities. However, the latter contain less information than the original Cg-identities [2,3,5]. Therefore, it could be possible that the original Cg-identities give stronger restrictions on the renormalizations (2.26-34) than (2.35-42). Fortunately this holds not true. Starting with an arbitrary renormalization (2.26-34) of the $\tilde{t}$ - (rsp. $t$-) distributions fulfilling (2.35-42), one can find a corresponding renormalization of the $t$-distributions which preserves the original $\mathrm{Cg}$-identities. This can be done in the following way: Let $\left.N\left(p_{1}, \ldots, p_{r}\right)\right|_{p_{1}+\ldots+p_{r}=0}$ be a renormalization polynomial of $\left.\tilde{t}_{B_{1} \ldots B_{r}}\left(p_{1}, \ldots, p_{r}, 0, \ldots, 0\right)\right|_{p_{1}+\ldots+p_{r}=0}$. This renormalization can be generated by the renormalization $t_{B_{1} \ldots B_{r}}\left(p_{1}, \ldots, p_{n-1}\right)+N\left(p_{1}, \ldots, p_{r}\right)$ and by keeping the other $t$-distributions (appearing in the definition of $\tilde{t}_{B_{1} \ldots B_{r}}$ ) unchanged. Together with a suitable renormalization of the $t_{u B_{2} \ldots B_{r}}^{l}$-distributions, $l=r+1, \ldots, n$, the original $\mathrm{Cg}$-identities can be maintained.

(B) By means of $Z_{i} \stackrel{\text { def }}{=} 1+\alpha_{i} C_{i}$ (where $\alpha_{i}$ is a suitable number, e.g. $\frac{g}{(2 \pi)^{2}}$ ) one can define finite Z-factors. Omitting terms quadratic in $C_{i}$, the equations (2.35-42) become the wellknown $[10,11,16]$ Z-factor relations. This is correct at one-loop level only. For the definition of the Z-factors in higher orders, the $C_{i}$ need to be normalization constants of one-particle irreducible diagrams. However, our $t, \tilde{t}$-distributions contain one-particle reducible terms. Therefore, and because the multiplicative renormalization of the Z-factors contrasts with our additive one (1.16), the Z-factors are not natural in our framework. It is well-known $[10,11,17]$ that the Slavnov-Taylor identities, which we are going to prove by means of our reduced $\mathrm{Cg}$-identities in the following section, imply the Z-factor relations in all orders.

\section{Derivation of the Slavnov-Taylor Identities from the reduced Cg-Identities}

The Slavnov-Taylor identities are given in terms of Green's functions (see some conventional textbook)

$$
<\Omega\left|T\left(\Phi_{j_{1} \text { int }}\left(x_{1}\right) \ldots \Phi_{j_{r} \text { int }}\left(x_{r}\right)\right)\right| \Omega>
$$


where $\Phi_{j_{1} \text { int }}\left(x_{1}\right), \ldots \Phi_{j_{r} \text { int }}\left(x_{r}\right)$ are interacting fields, $T$ means the time ordering and $\mid \Omega>$ is the vacuum or ground state of the interacting theory. By means of the Gell-Mann Low series [13] we know the perturbative expansion of (3.1) (see the examples below): Let $k_{1}, k_{2}, \ldots, k_{r}$ be the external momenta, where $k_{1}+k_{2}+\ldots+k_{r}=0$. In order to avoid infrared divergences, we always assume all $k_{I} \stackrel{\text { def }}{=} \sum_{i \in I} k_{i}$ to be off-shell (where $I$ runs through all subsets of $\{1,2, . ., r\}$ with $1,2, \ldots, r-1$ elements). Then, as we will see, our assumption (1.24-25) implies the existence of the Gell-Mann Low expressions in the following sense: Every point $k \stackrel{\text { def }}{=}\left(k_{1}, \ldots, k_{r-1}\right)$ in the above region has a neighbourhood $U_{k} \subset \mathbf{R}^{4(r-1)}$, such that the GellMann Low expressions are distributions on the space of testfunctions with support in $U_{k}$. Because of the degenerate terms it does not suffice to keep only $k_{1}, k_{2}, \ldots, k_{r}$ off-shell. For our purposes we only need the connected Green's functions $<\Omega\left|T\left(\Phi_{j_{1} \text { int }}\left(x_{1}\right) \ldots \Phi_{j_{r} \text { int }}\left(x_{r}\right)\right)\right|$ $\Omega>_{c}$. Their perturbative expansion is obtained by omitting the disconnected diagrams in the Gell-Mann Low series. This omission is no problem for us, since our $t, \tilde{t}, \tau$-distributions contain connected diagrams only.

Epstein and Glaser [6] give another definition of Green's functions in the framework of causal perturbation theory. In these expressions the inner vertices are smeared out with $g \in \mathcal{S}\left(\mathbf{R}^{4}\right)$. Due to Epstein and Glaser [6] the adiabatic limit $g \rightarrow 1$ exists for massive theories. In this limit their Green's functions agree with the Gell-Mann Low expressions, if the latter are infrared-improved in an appropriate way. This is proven in the appendix for the massive case. In our Yang-Mills model with matter fields the infrared behaviour is worse [23]. Therefore, we only consider the region with all $k_{I}$ (defined above) off-shell. In this region the two definitions of Green's functions probably agree if the adiabatic limit is taken in the Epstein-Glaser expressions. In fact, in the appendix it is demonstrated by an explicit calculation that this is true at least for the 2-point Green's functions in lowest non-trivial order. Since the aim of this paper is to derive the usual Slavnov-Taylor identities from our Cg-identities, we work with the conventional perturbative expansion of Green's functions the (renormalized) Gell-Mann Low series for (3.1).

\section{(a) Two-Legs Slavnov-Taylor Identity}

For the sake of completeness we summarize and discuss sect.3.3 of [2]. One defines the 2-point Green's function or gluon propagator [16] by

$$
i D_{a b}^{\mu \nu}(k) \equiv i \delta_{a b} D^{\mu \nu}(k) \stackrel{\text { def }}{=}(2 \pi)^{-2} \int d^{4} x e^{i k x}<\Omega\left|T\left(A_{\text {int } a}^{\mu}(x) A_{\text {int } b}^{\nu}(0)\right)\right| \Omega>_{c}
$$

where $A_{\text {int }}$ is the interacting gluon field. (3.2) has the following perturbative expansion

$$
i D_{a b}^{\mu \nu}(k)=-i(2 \pi)^{-2} \delta_{a b} \frac{g^{\mu \nu}}{k^{2}+i 0}-\delta_{a b} \frac{1}{k^{2}+i 0} \sum_{n=1}^{\infty} \frac{(2 \pi)^{(4 n-4)}}{(2 n-2) !} \tilde{t}_{A A}^{(2 n) \mu \nu}(k,-k, 0, \ldots, 0) \frac{1}{k^{2}+i 0}
$$

where $\tilde{t}_{A A}^{(2 n)}$ is defined in (2.1). Besides the limit (1.25) of the $\hat{t}$-distributions, there is a second source of infrared divergences in the Gell-Mann Low series, namely the square of a Feynman propagator, which appears in the two-legs terms. Without an infrared regularization (e.g. the adiabatic switching with $g \in \mathcal{S}\left(\mathbf{R}^{4}\right)$, see the appendix), the terms $n \geq 1$ in (3.3) do not exist (in the sense of distributions in $k$ ) in a neighbourhood of $k^{2}=0$, except the normalization constants $C_{A A}^{(2 n)}(2.35)$ of $\tilde{t}_{A A}^{(2 n)}$ can be chosen in such a way that

$$
\tilde{t}_{A A}^{(2 n) \mu \nu}(k,-k, 0, \ldots, 0) \sim k^{2}\left(k^{\mu} k^{\nu}-g^{\mu \nu} k^{2}\right), \quad \forall n
$$


for $k^{2} \rightarrow 0$. Such a normalization is given in QED by the central solution of the distribution splitting $[6,8,18]$ and it exists also for the matter loop in $\tilde{t}_{A A}^{(2)}$ (second order) if the matter fields are massive. However, the sum of the gluon and ghost loop is [2]

$$
\sim\left(k^{\mu} k^{\nu}-g^{\mu \nu} k^{2}\right) \log \frac{-\left(k^{2}+i 0\right)}{M^{2}}
$$

in second order, where $M$ is an arbitrary constant. (The choice of $M$ is the choice of $C_{A A}^{(2)}$ (2.35).) Obviously (3.3a) is impossible. We avoid serious problems by assuming throughout all (sums of) external momenta to be off-shell.

By means of (2.5) we obtain the well-known Slavnov-Taylor identity $[11,16]$

$$
k_{\mu} D_{a b}^{\mu \nu}(k)=-(2 \pi)^{-2} \delta_{a b} \frac{k^{\nu}}{k^{2}}
$$

and this is the only one with two legs.

\section{(b) Three-Legs Slavnov-Taylor Identities}

Analogous to (3.1) one defines [16] the ghost propagator by

$$
i \tilde{D}_{a b}(k) \equiv i \delta_{a b} \tilde{D}(k): \stackrel{\text { def }}{=}(2 \pi)^{-2} \int d^{4} x e^{i k x}<\Omega\left|T\left(u_{\operatorname{int} a}(x) \tilde{u}_{\text {int } b}(0)\right)\right| \Omega>_{c}
$$

and the matter propagator by

$$
i S_{\alpha \beta}(p) \equiv i \delta_{\alpha \beta} S(p): \stackrel{\text { def }}{=}(2 \pi)^{-2} \int d^{4} x e^{i p x}<\Omega\left|T\left(\psi_{\operatorname{int} \alpha}(x) \bar{\psi}_{\operatorname{int} \beta}(0)\right)\right| \Omega>_{c} .
$$

Their perturbative expansions read in terms of our $\hat{t}$-distributions

$$
\begin{gathered}
i \tilde{D}_{a b}(k)=\frac{i}{(2 \pi)^{2}} \frac{\delta_{a b}}{k^{2}}+\delta_{a b} \frac{i k_{\mu}}{k^{2}} \sum_{n=1}^{\infty} \frac{(2 \pi)^{(4 n-4)}}{(2 n-2) !} t_{u \tilde{u}}^{\mu(2 n)}(-k, k, 0, \ldots, 0) \frac{1}{k^{2}}, \quad k^{2} \neq 0, \\
i S_{\alpha \beta}(p)=\frac{i}{(2 \pi)^{2}} \delta_{\alpha \beta} \frac{\gamma^{\nu} p_{\nu}+m}{p^{2}-m^{2}}- \\
-\delta_{\alpha \beta} \frac{\gamma^{\nu} p_{\nu}+m}{p^{2}-m^{2}} \sum_{n=1}^{\infty} \frac{(2 \pi)^{(4 n-4)}}{(2 n-2) !} \tau_{\bar{\psi} \psi}^{(2 n)}(p,-p, 0, \ldots, 0) \frac{\gamma^{\nu} p_{\nu}+m}{p^{2}-m^{2}}, \quad p^{2} \neq m^{2} .
\end{gathered}
$$

Note that $\tilde{D}(k)(3.5)$ has the structure

$$
\tilde{D}(k)=\tilde{D}_{0}(k)+k_{\mu} \tilde{D}_{1}^{\mu}(k), \quad \text { where } \quad \tilde{D}_{0}(k) \stackrel{\text { def }}{=} \frac{1}{(2 \pi)^{2}} \frac{1}{k^{2}}
$$

is the contribution of zeroth order. The factor $k_{\mu}$ in $k_{\mu} \tilde{D}_{1}^{\mu}(k)$ originates from the derivative $\partial_{\mu}$ in $t_{u \tilde{u}}^{\mu}: u \partial_{\mu} \tilde{u}:$. The covariant decomposition of $\tilde{D}_{1}^{\mu}(k)(3.9)$ is

$$
\tilde{D}_{1}^{\mu}(k)=k^{\mu} \tilde{D}_{2}(k) .
$$


Now we will consider the 3-point Green's functions corresponding to the couplings $T_{1}^{A}, T_{1}^{u}$ and $T_{1}^{\psi}$. We are going to define the connected Green's functions $\tilde{T}, \tilde{G}, \tilde{\Gamma}$ and the proper (or one-particle irreducible) vertex functions $T, G, \Gamma$ which are the connected Green's functions with amputated legs

$$
\begin{gathered}
\left.\quad i g T_{a b c}^{\mu^{\prime} \nu^{\prime} \tau^{\prime}}(p, q, k) i D_{\mu^{\prime}}^{\mu}(p) i D_{\nu^{\prime}}^{\nu}(q) i D_{\tau^{\prime}}^{\tau}(k)\right|_{p+q+k=0}:\left.\stackrel{\text { def }}{=} \tilde{T}_{a b c}^{\mu \nu \tau}(p, q, k)\right|_{p+q+k=0}: \stackrel{\text { def }}{=} \\
\stackrel{\text { def }}{=}(2 \pi)^{-4} \int d^{4} x \int d^{4} y e^{i p x} e^{i q y}<\Omega\left|T\left(A_{\operatorname{int} a}^{\mu}(x) A_{\operatorname{int} b}^{\nu}(y) A_{\operatorname{int} c}^{\tau}(0)\right)\right| \Omega>_{c} \\
\left.\quad i g G_{a b c}^{\mu^{\prime}}(k, q, p) i D_{\mu^{\prime}}^{\mu}(k) i \tilde{D}(q) i \tilde{D}(p)\right|_{p+q+k=0}:\left.\stackrel{\text { def }}{=} \tilde{G}_{a b c}^{\mu}(k, q, p)\right|_{p+q+k=0}: \stackrel{\text { def }}{=} \\
\stackrel{\text { def }}{=}(2 \pi)^{-4} \int d^{4} x \int d^{4} y e^{i p x} e^{i q y}<\Omega\left|T\left(u_{\operatorname{int} c}(x) \tilde{u}_{\operatorname{int} b}(y) A_{\operatorname{int} a}^{\mu}(0)\right)\right| \Omega>_{c}
\end{gathered}
$$

and

$$
\begin{gathered}
\left.i S(p) i g \Gamma_{\alpha \beta a}^{\mu^{\prime}}(p, q, k) i S(-q) i D_{\mu^{\prime}}^{\mu}(k)\right|_{p+q+k=0}:\left.\stackrel{\text { def }}{=} \tilde{\Gamma}_{\alpha \beta a}^{\mu}(p, q, k)\right|_{p+q+k=0}: \stackrel{\text { def }}{=} \\
\stackrel{\text { def }}{=}(2 \pi)^{-4} \int d^{4} x \int d^{4} y e^{i p x} e^{i q y}<\Omega\left|T\left(\psi_{\operatorname{int} \alpha}(x) \bar{\psi}_{\operatorname{int} \beta}(y) A_{\operatorname{int} a}^{\mu}(0)\right)\right| \Omega>_{c} .
\end{gathered}
$$

By means of the Gell-Mann Low series we can express the perturbative expansions of $\tilde{T}, \tilde{\Gamma}$ and $\tilde{G}$ in terms of our $t$-distributions. The results in $n$-th order $(n \geq 3)$ contain nondegenerate terms $\sim \tilde{t}_{B_{1} B_{2} B_{3}}^{(n)}$ and degenerate ones which consist of a two-legs distribution in order $(n-1)$ and one further vertex (fig.2). We only consider the region $p+q+k=0, p^{2} \neq 0$ rsp. $p^{2} \neq m^{2}, q^{2} \neq 0$ rsp. $q^{2} \neq m^{2}, k^{2} \neq 0$

$$
\begin{gathered}
\left.\tilde{T}_{a b c}^{(n) \mu \nu \tau}(p, q, k)\right|_{p+q+k=0}=\frac{(2 \pi)^{(2 n-6)} i f_{a b c}}{(n-3) ! p^{2} q^{2} k^{2}}\left\{\tilde{t}_{A A A}^{(n) \mu \nu \tau}(p, q, k, 0, \ldots)+\right. \\
+\frac{i g}{(2 \pi)^{2}}\left[\tilde{t}_{A A}^{(n-1) \mu \mu^{\prime}}(p,-p, 0, \ldots) \frac{1}{p^{2}}\left[g_{\mu^{\prime}}^{\nu}(p-q)^{\tau}+g^{\nu \tau}(q-k)_{\mu^{\prime}}+g_{\mu^{\prime}}^{\tau}(k-p)^{\nu}\right]+\right. \\
+\tilde{t}_{A A}^{(n-1) \nu \nu^{\prime}}(q,-q, 0, \ldots) \frac{1}{q^{2}}\left[g_{\nu^{\prime}}^{\mu}(p-q)^{\tau}+g_{\nu^{\prime}}(q-k)^{\mu}+g^{\mu \tau}(k-p)_{\nu^{\prime}}\right]+ \\
\left.\left.+\tilde{t}_{A A}^{(n-1) \tau \tau^{\prime}}(k,-k, 0, \ldots) \frac{1}{k^{2}}\left[g^{\mu \nu}(p-q)_{\tau^{\prime}}+g_{\tau^{\prime}}^{\nu}(q-k)^{\mu}+g_{\tau^{\prime}}^{\mu}(k-p)^{\nu}\right]\right]\right\}\left.\right|_{p+q+k=0}, \\
\left.\tilde{\Gamma}_{\alpha \beta a}^{(n) \mu}(p, q, k)\right|_{p+q+k=0}=\frac{(2 \pi)^{(2 n-6)} i}{(n-3) ! k^{2}}\left(\lambda_{a}\right)_{\alpha \beta} \frac{\gamma^{\nu} p_{\nu}+m}{p^{2}-m^{2}}\left\{\tilde{\tau}_{\bar{\psi} \psi A}^{(n) \mu}(p, q, k, 0, \ldots)+\right. \\
+\frac{g}{2(2 \pi)^{2}}\left[\gamma_{\lambda} \frac{1}{k^{2}} \tilde{t}_{A A}^{(n-1) \mu \lambda}(k,-k, 0, \ldots)-\gamma^{\mu} \frac{-\gamma^{\nu} q_{\nu}+m}{q^{2}-m^{2}} \tau_{\bar{\psi} \psi}^{(n-1)}(-q, q, 0, \ldots)-\right. \\
\left.\left.-\tau_{\bar{\psi} \psi}^{(n-1)}(p,-p, 0, \ldots) \frac{\gamma^{\nu} p_{\nu}+m}{p^{2}-m^{2}} \gamma^{\mu}\right]\right\}\left.\frac{-\gamma^{\nu} q_{\nu}+m}{q^{2}-m^{2}}\right|_{p+q+k=0}
\end{gathered}
$$

and

$$
\left.\tilde{G}_{a b c}^{\mu}(k, q, p)\right|_{p+q+k=0}=\left.\tilde{G}_{1 a b c}^{\mu \lambda}(k, q, p) p_{\lambda}\right|_{p+q+k=0}
$$

with

$$
\left.\tilde{G}_{1 a b c}^{(n) \mu \lambda}(k, q, p)\right|_{p+q+k=0}=\frac{(2 \pi)^{(2 n-6)} f_{a b c}}{(n-3) ! p^{2} q^{2} k^{2}}\left\{\tilde{t}_{A u \tilde{u}}^{(n) \mu \lambda}(k, q, p, 0, \ldots)+\right.
$$


$\left.+\frac{g}{(2 \pi)^{2}}\left[-\tilde{t}_{A A}^{(n-1) \mu \lambda}(k,-k, 0, \ldots) \frac{1}{k^{2}}+g^{\mu \lambda} \frac{i q_{\tau}}{q^{2}} t_{u \tilde{u}}^{\tau}(q,-q, 0, \ldots)-t_{u \tilde{u}}^{\lambda}(-p, p, 0, \ldots) \frac{i p^{\mu}}{p^{2}}\right]\right\}\left.\right|_{p+q+k=0}$.

Similar to (3.9) the factor $p_{\lambda}$ in (3.16) comes from $\partial_{\lambda} \tilde{u}$ which is contracted with the external vertex $u_{c}(x)$ in $<\Omega\left|T\left(u_{\operatorname{int} c}(x) \ldots\right)\right| \Omega>_{c}$. Note that the proper vertex $G_{a b c}^{\mu}(k, q, p)(3.12)$ has an external leg $\partial_{\nu} \tilde{u}$ and that the corresponding factor $p_{\nu}$ is absorbed in $G^{\mu}$

$$
\left.G_{a b c}^{\mu}(k, q, p)\right|_{p+q+k=0}=\left.G_{1 a b c}^{\mu \nu}(k, q, p) p_{\nu}\right|_{p+q+k=0} .
$$

Now we insert (3.9-10) into $\tilde{D}(p)$ in (3.12). Moreover we use (3.16), (3.18) there and omit the factor $p_{\lambda}$ coming from the contraction of $\partial_{\lambda} \tilde{u}$ with the external vertex $u_{c}(x)$. It results

$$
\left.\tilde{G}_{1 a b c}^{\mu \lambda}(k, q, p)\right|_{p+q+k=0}=\left.g G_{1 a b c}^{\mu^{\prime} \nu}(k, q, p) D_{\mu^{\prime}}^{\mu}(k) \tilde{D}(q)\left(\tilde{D}_{0}(p) g_{\nu}{ }^{\lambda}+p_{\nu} p^{\lambda} \tilde{D}_{2}(p)\right)\right|_{p+q+k=0} .
$$

Let us consider the Slavnov-Taylor identity (formula (1.1) of [19] or (2.8) of [17] in the Feynman gauge)

$$
\begin{gathered}
\left.\frac{p_{1 \alpha}}{p_{1}^{2}} T_{a b c}^{\alpha \mu^{\prime} \nu^{\prime}}\left(p_{1}, p_{2}, p_{3}\right)\right|_{p_{1}+p_{2}+p_{3}=0}=\left(D_{\lambda}^{\nu^{\prime}-1}\left(p_{3}\right)+(2 \pi)^{2} p_{3}^{\nu^{\prime}} p_{3 \lambda}\right) G_{1 \text { bac }}^{\mu^{\prime} \lambda}\left(p_{2}, p_{1}, p_{3}\right) \tilde{D}\left(p_{1}\right)+ \\
+\left.\left(D_{\lambda}^{\mu^{\prime}-1}\left(p_{2}\right)+(2 \pi)^{2} p_{2}^{\mu^{\prime}} p_{2 \lambda}\right) G_{1 c a b}^{\nu^{\prime} \lambda}\left(p_{3}, p_{1}, p_{2}\right) \tilde{D}\left(p_{1}\right)\right|_{p_{1}+p_{2}+p_{3}=0}
\end{gathered}
$$

which is written in terms of one-particle irreducible Green's functions and $p_{i}^{2} \neq 0, i=1,2,3$ is assumed. We multiply (3.20) with $D_{\mu^{\prime}}^{\mu}\left(p_{2}\right) D_{\nu^{\prime}}^{\nu}\left(p_{3}\right)$ and use (3.4). It results the equivalent equation

$$
\begin{gathered}
\frac{p_{1 \alpha}}{p_{1}^{2}} T_{a b c}^{\alpha \mu^{\prime} \nu^{\prime}}\left(p_{1}, p_{2}, p_{3}\right) D_{\mu^{\prime}}^{\mu}\left(p_{2}\right) D_{\nu^{\prime}}^{\nu}\left(p_{3}\right)=D_{\mu^{\prime}}^{\mu}\left(p_{2}\right) \frac{1}{p_{3}^{2}}\left(g_{\lambda}^{\nu} p_{3}^{2}-p_{3}^{\nu} p_{3 \lambda}\right) G_{1 \text { bac }}^{\mu^{\prime} \lambda}\left(p_{2}, p_{1}, p_{3}\right) \tilde{D}\left(p_{1}\right)+ \\
+D_{\nu^{\prime}}^{\nu}\left(p_{3}\right) \frac{1}{p_{2}^{2}}\left(g_{\lambda}^{\mu} p_{2}^{2}-p_{2}^{\mu} p_{2 \lambda}\right) G_{1 \text { cab }}^{\nu^{\prime} \lambda}\left(p_{3}, p_{1}, p_{2}\right) \tilde{D}\left(p_{1}\right) .
\end{gathered}
$$

By means of (3.4) the l.h.s. can be expressed by $\tilde{T}$ (3.11). With (3.19) the first term on the r.h.s. reads in terms of $\tilde{G}(3.12)$ as follows

$$
G_{1 b a c}^{\mu^{\prime} \lambda}\left(p_{2}, p_{1}, p_{3}\right) D_{\mu^{\prime}}^{\mu}\left(p_{2}\right) \tilde{D}\left(p_{1}\right) \tilde{D}_{0}\left(p_{3}\right)\left(g_{\lambda}^{\nu} p_{3}^{2}-p_{3}^{\nu} p_{3 \lambda}\right)=\frac{1}{g} \tilde{G}_{1 b a c}^{\mu \lambda}\left(p_{2}, p_{1}, p_{3}\right)\left(g_{\lambda}^{\nu} p_{3}^{2}-p_{3}^{\nu} p_{3 \lambda}\right) .
$$

Summing up the Slavnov-Taylor identity (3.20) is equivalent to

$$
\begin{gathered}
-\left.p_{1 \alpha} \tilde{T}_{a b c}^{\alpha \mu \nu}\left(p_{1}, p_{2}, p_{3}\right)\right|_{p_{1}+p_{2}+p_{3}=0}= \\
=\tilde{G}_{1 b a c}^{\mu \lambda}\left(p_{2}, p_{1}, p_{3}\right)\left(g_{\lambda}^{\nu} p_{3}^{2}-p_{3}^{\nu} p_{3 \lambda}\right)+\left.\tilde{G}_{1 c a b}^{\nu \lambda}\left(p_{3}, p_{1}, p_{2}\right)\left(g_{\lambda}^{\mu} p_{2}^{2}-p_{2}^{\mu} p_{2 \lambda}\right)\right|_{p_{1}+p_{2}+p_{3}=0},
\end{gathered}
$$

which is an identity between the connected Green's functions. (Slavnov's identities [11] are originally written in terms of connected Green's functions. For someone who is very familiar with Slavnov's formalism it may therefore be easier to write Slavnov's identity directly in the form (3.23), without the detour (3.20) with the one-particle irreducible Green's functions. (3.20) rsp. (3.23) cannot be obtained from 't Hooft's diagramatic Ward identities [12] or from Taylor's identities [10], because in these identities some of the external momenta are on-shell.) 
Now we are going to show that the perturbative version of (3.23) is essentially our reduced $C g$-identity (2.14). For this purpose we insert the $n$-th order expressions (3.14) and (3.17). By means of the 2-legs Cg-identity (2.5) we obtain for the 1.h.s. of (3.23)

$$
\begin{gathered}
\text { l.h.s. }{ }^{(n)}=\frac{(2 \pi)^{(2 n-6)} f_{a b c}}{(n-3) ! p_{1}^{2} p_{2}^{2} p_{3}^{2}}\left\{-i p_{1 \alpha} \tilde{t}_{A A A}^{\alpha \mu \nu}\left(p_{1}, p_{2}, p_{3}, 0, \ldots\right)+\right. \\
+\frac{g}{(2 \pi)^{2}}\left[\tilde{t}_{A A}^{\mu \mu^{\prime}}\left(p_{2},-p_{2}, 0, \ldots\right) \frac{1}{p_{2}^{2}}\left[-p_{1 \mu^{\prime}} p_{2}^{\nu}+g_{\mu^{\prime}}^{\nu} p_{1}\left(p_{2}-p_{3}\right)+p_{1}^{\nu} p_{3 \mu^{\prime}}\right]+\right. \\
\left.\left.+\tilde{t}_{A A}^{\nu \nu^{\prime}}\left(p_{3},-p_{3}, 0, \ldots\right) \frac{1}{p_{3}^{2}}\left[-p_{1}^{\mu} p_{2 \nu^{\prime}}+g_{\nu^{\prime}}^{\mu} p_{1}\left(p_{2}-p_{3}\right)+p_{1 \nu^{\prime}} p_{3}^{\mu}\right]\right]\right\}\left.\right|_{1}+p_{2}+p_{3}=0= \\
=\frac{(2 \pi)^{(2 n-6)} f_{a b c}}{(n-3) ! p_{1}^{2} p_{2}^{2} p_{3}^{2}}\left\{-i p_{1 \alpha} \tilde{t}_{A A A}^{\alpha \mu \nu}\left(p_{1}, p_{2}, p_{3}, 0, \ldots\right)+\right. \\
+\frac{g}{(2 \pi)^{2}}\left[\tilde{t}_{A A}^{\mu \nu}\left(-p_{3}, p_{3}, 0, \ldots\right)-\tilde{t}_{A A}^{\nu \mu}\left(-p_{2}, p_{2}, 0, \ldots\right)+\right. \\
\left.\left.+\tilde{t}_{A A}^{\mu \mu^{\prime}}\left(p_{2},-p_{2}, 0, \ldots\right) \frac{1}{p_{2}^{2}}\left(g_{\mu^{\prime}}^{\nu} p_{3}^{2}-p_{3 \mu^{\prime}} p_{3}^{\nu}\right)-\tilde{t}_{A A}^{\nu \nu^{\prime}}\left(p_{3},-p_{3}, 0, \ldots\right) \frac{1}{p_{3}^{2}}\left(g_{\nu^{\prime}}^{\mu} p_{2}^{2}-p_{2 \nu^{\prime}} p_{2}^{\mu}\right)\right]\right\}\left.\right|_{p_{1}+p_{2}+p_{3}=0}
\end{gathered}
$$

Using the covariant decomposition (2.4) of $t_{u \tilde{u}}^{\mu}$, the r.h.s. of (3.23) reads

$$
\begin{gathered}
\text { r.h.s. }{ }^{(n)}=\frac{(2 \pi)^{(2 n-6)} f_{a b c}}{(n-3) ! p_{1}^{2} p_{2}^{2} p_{3}^{2}}\left\{-\tilde{t}_{A u \tilde{u}}^{\mu \lambda}\left(p_{2}, p_{1}, p_{3}, 0, \ldots\right)\left(g_{\lambda}^{\nu} p_{3}^{2}-p_{3}^{\nu} p_{3 \lambda}\right)+\right. \\
+\tilde{t}_{A u \tilde{u}}^{\nu \lambda}\left(p_{3}, p_{1}, p_{2}, 0, \ldots\right)\left(g_{\lambda}^{\mu} p_{2}^{2}-p_{2}^{\mu} p_{2 \lambda}\right)+\frac{g}{(2 \pi)^{2}}\left[i \bar{t}_{u \tilde{u}}\left(p_{1}^{2}\right)\left[-p_{3}^{2} g^{\mu \nu}+p_{3}^{\mu} p_{3}^{\nu}+p_{2}^{2} g^{\mu \nu}-p_{2}^{\mu} p_{2}^{\nu}\right]+\right. \\
\left.\left.+\tilde{t}_{A A}^{\mu \mu^{\prime}}\left(p_{2},-p_{2}, 0, \ldots\right) \frac{1}{p_{2}^{2}}\left(g_{\mu^{\prime}}^{\nu} p_{3}^{2}-p_{3 \mu^{\prime}} p_{3}^{\nu}\right)-\tilde{t}_{A A}^{\nu \nu^{\prime}}\left(p_{3},-p_{3}, 0, \ldots\right) \frac{1}{p_{3}^{2}}\left(g_{\nu^{\prime}}^{\mu} p_{2}^{2}-p_{2 \nu^{\prime}} p_{2}^{\mu}\right)\right]\right\}\left.\right|_{p_{1}+p_{2}+p_{3}=0} .
\end{gathered}
$$

The equality $(3.24)=(3.25)$ is exactly our reduced Cg-identity (2.14). This proves the Slavnov-Taylor identity (3.23) in the framework of causal perturbation theory.

(3.20) rsp. (3.23) is the only Slavnov-Taylor identity with three external legs in pure Yang-Mills theories [10,11]. If we add the coupling to matter fields, there is precisely one additional identity of this kind [10], which corresponds to the reduced Cg-identity (2.9). In order to formulate this identity, Taylor is forced to introduce the Q-vertex [10]. (This is in accordance with the fact that the Q-vertex cannot be eliminated completely in (2.9).) We define the connected Green's functions $S^{F}(p) \gamma^{\mu} \tilde{\Gamma}^{1}(p, q, k)$ and $\tilde{\Gamma}^{2}(p, q, k) \gamma^{\mu} S^{F}(-q)$

$$
\begin{gathered}
\left.S^{F}(p) \gamma^{\mu} \tilde{\Gamma}_{\alpha \beta a}^{1}(p, q, k)\right|_{p+q+k=0}: \stackrel{\text { def }}{=} \frac{-g}{2(2 \pi)^{4}} \int d^{4} x \int d^{4} y e^{i p x} e^{i q y} \int d^{4} x_{1} \\
<\Omega\left|T\left(S^{F}\left(x-x_{1}\right)\left(\lambda_{a^{\prime}}\right)_{\alpha \alpha^{\prime}} \gamma^{\mu} \psi_{\operatorname{int} \alpha^{\prime}}\left(x_{1}\right) u_{\operatorname{int} a^{\prime}}\left(x_{1}\right) \bar{\psi}_{\operatorname{int} \beta}(y) \tilde{u}_{\operatorname{int} a}(0)\right)\right| \Omega>_{c}, \\
\left.\tilde{\Gamma}_{\alpha \beta a}^{2}(p, q, k) \gamma^{\mu} S^{F}(-q)\right|_{p+q+k=0}: \stackrel{\text { def }}{=} \frac{-g}{2(2 \pi)^{4}} \int d^{4} x \int d^{4} y e^{i p x} e^{i q y} \int d^{4} x_{1} \\
<\Omega\left|T\left(\psi_{\operatorname{int} \alpha}(x) \bar{\psi}_{\operatorname{int} \beta^{\prime}}\left(x_{1}\right)\left(\lambda_{a^{\prime}}\right)_{\beta^{\prime} \beta} \gamma^{\mu} S^{F}\left(x_{1}-y\right) u_{\text {int } a^{\prime}}\left(x_{1}\right) \tilde{u}_{\text {int } a}(0)\right)\right| \Omega>_{c} .
\end{gathered}
$$


They have one Q-vertex at $x_{1}$ which is directly contracted with $\psi_{\alpha}(x)$ rsp. $\bar{\psi}_{\beta}(y)$, all other vertices are ordinary vertices. By means of the Gell-Mann Low series we obtain

$$
\begin{gathered}
\left.\tilde{\Gamma}_{\alpha \beta a}^{(n) 1}(p, q, k)\right|_{p+q+k=0}=\frac{(2 \pi)^{(2 n-4)} i}{(n-3) ! k^{2}}\left(\lambda_{a}\right)_{\alpha \beta}\left\{\bar{\tau}_{\bar{\psi} \psi u}^{(n) 1}(p, q, k, 0, \ldots)+\right. \\
\left.+\frac{g}{2(2 \pi)^{2}}\left[-i t_{u \tilde{u}}^{(n-1) \tau}(k,-k, 0, \ldots) \frac{k_{\tau}}{k^{2}}-\frac{-\gamma^{\nu} q_{\nu}+m}{q^{2}-m^{2}} \tau_{\bar{\psi} \psi}^{(n-1)}(-q, q, 0, \ldots)\right]\right\}\left.\frac{-\gamma^{\nu} q_{\nu}+m}{q^{2}-m^{2}}\right|_{p+q+k=0}, \\
\left.\tilde{\Gamma}_{\alpha \beta a}^{(n) 2}(p, q, k)\right|_{p+q+k=0}=\frac{(2 \pi)^{(2 n-4)}}{(n-3) ! k^{2}}\left(\lambda_{a}\right)_{\alpha \beta} \frac{\gamma^{\nu} p_{\nu}+m}{p^{2}-m^{2}}\left\{\bar{\tau}_{\bar{\psi} \psi u}^{(n) 2}(p, q, k, 0, \ldots)+\right. \\
\left.+\frac{g}{2(2 \pi)^{2}}\left[-i t_{u \tilde{u}}^{(n-1) \tau}(k,-k, 0, \ldots) \frac{k_{\tau}}{k^{2}}-\tau_{\bar{\psi} \psi}^{(n-1)}(p,-p, 0, \ldots) \frac{\gamma^{\nu} p_{\nu}+m}{p^{2}-m^{2}}\right]\right\}\left.\right|_{p+q+k=0},
\end{gathered}
$$

in $n$-th order perturbation theory $(n \geq 3)$ and $p^{2} \neq m^{2}, q^{2} \neq m^{2}, k^{2} \neq 0$ is assumed. In terms of connected Green's functions Taylor's identity reads

$$
\begin{gathered}
-\left.p_{3 \mu} \tilde{\Gamma}_{\alpha \beta a}^{\mu}\left(p_{1}, p_{2}, p_{3}\right)\right|_{p_{1}+p_{2}+p_{3}=0}= \\
=S^{F}\left(p_{1}\right)\left(\gamma^{\mu} p_{1 \mu}-m\right) \tilde{\Gamma}_{\alpha \beta a}^{1}\left(p_{1}, p_{2}, p_{3}\right)+\left.\tilde{\Gamma}_{\alpha \beta a}^{2}\left(p_{1}, p_{2}, p_{3}\right)\left(\gamma^{\mu} p_{2 \mu}+m\right) S^{F}\left(-p_{2}\right)\right|_{p_{1}+p_{2}+p_{3}=0} \equiv \\
\left.\equiv(2 \pi)^{-2}\left[\tilde{\Gamma}_{\alpha \beta a}^{1}\left(p_{1}, p_{2}, p_{3}\right)-\tilde{\Gamma}_{\alpha \beta a}^{2}\left(p_{1}, p_{2}, p_{3}\right)\right]\right|_{p_{1}+p_{2}+p_{3}=0},
\end{gathered}
$$

where the external momenta are off-shell. In order to obtain Taylor's original identity (formula (14) of [10]) from (3.30), one has to express $\tilde{\Gamma}(3.13), \tilde{\Gamma}^{1}, \tilde{\Gamma}^{2}$ by proper vertices and by 2-point Green's functions, and to amputate the three external Feynman propagators. Then, one must multiply by $\bar{u}\left(p_{1}\right)$, which eliminates the $\tilde{\Gamma}^{1}$-term on the r.h.s.. This multiplication puts $p_{1}$ on the mass-shell $\bar{u}\left(p_{1}\right) \gamma^{\nu} p_{1 \nu}=\bar{u}\left(p_{1}\right) m$ and, therefore, infrared divergences appear.

Let us consider (3.30) in perturbation theory. We insert the $n$-th order expressions (3.15), (3.28) and (3.29). With the 2-legs Cg-identity (2.5) the l.h.s. of (3.30) becomes

$$
\begin{gathered}
\text { l.h.s. }{ }^{(n)}=\frac{(2 \pi)^{(2 n-6)} i}{(n-3) ! p_{3}^{2}}\left(\lambda_{a}\right)_{\alpha \beta} \frac{\gamma^{\nu} p_{1 \nu}+m}{p_{1}^{2}-m^{2}}\left\{-p_{3 \mu} \tilde{\tau}_{\bar{\psi} \psi A}^{(n) \mu}\left(p_{1}, p_{2}, p_{3}, 0, \ldots\right)+\right. \\
+\frac{g}{2(2 \pi)^{2}}\left[\tau_{\bar{\psi} \psi}^{(n-1)}\left(-p_{2}, p_{2}, 0, \ldots\right)-\tau_{\bar{\psi} \psi}^{(n-1)}\left(p_{1},-p_{1}, 0, \ldots\right)+\right. \\
+\left(\gamma^{\mu}\left(p_{3}+p_{2}\right)_{\mu}+m\right) \frac{-\gamma^{\nu} p_{2 \nu}+m}{p_{2}^{2}-m^{2}} \tau \tau_{\bar{\psi} \psi}^{(n-1)}\left(-p_{2}, p_{2}, 0, \ldots\right)+ \\
\left.\left.+\tau_{\bar{\psi} \psi}^{(n-1)}\left(p_{1},-p_{1}, 0, \ldots\right) \frac{\gamma^{\nu} p_{1 \nu}+m}{p_{1}^{2}-m^{2}}\left(\gamma^{\mu}\left(p_{3}+p_{1}\right)_{\mu}-m\right)\right]\right\}\left.\frac{-\gamma^{\nu} p_{2 \nu}+m}{p_{2}^{2}-m^{2}}\right|_{p_{1}+p_{2}+p_{3}=0 .} .
\end{gathered}
$$

Using the covariant decomposition (2.4) of $t_{u \tilde{u}}^{\mu}$, we obtain for the r.h.s.

$$
\begin{gathered}
\text { r.h.s. }{ }^{(n)}=\frac{(2 \pi)^{(2 n-6)} i}{(n-3) ! p_{3}^{2}}\left(\lambda_{a}\right)_{\alpha \beta} \frac{\gamma^{\nu} p_{1 \nu}+m}{p_{1}^{2}-m^{2}}\left\{\left(\gamma^{\mu} p_{1 \mu}-m\right) \bar{\tau}_{\bar{\psi} \psi u}^{(n) 1}\left(p_{1}, p_{2}, p_{3}, 0, \ldots\right)+\right. \\
+\bar{\tau}_{\bar{\psi} \psi u}^{(n) 2}\left(p_{1}, p_{2}, p_{3}, 0, \ldots\right)\left(\gamma^{\mu} p_{2 \mu}+m\right)+\frac{g}{2(2 \pi)^{2}}\left[i \gamma_{\mu} t_{u \tilde{u}}^{(n-1) \mu}\left(p_{3},-p_{3}, 0, \ldots\right)+\right. \\
+\left(-\gamma^{\mu} p_{1 \mu}+m\right) \frac{-\gamma^{\nu} p_{2 \nu}+m}{p_{2}^{2}-m^{2}} \tau_{\bar{\psi} \psi}^{(n-1)}\left(-p_{2}, p_{2}, 0, \ldots\right)-
\end{gathered}
$$




$$
\left.\left.-\tau_{\bar{\psi} \psi}^{(n-1)}\left(p_{1},-p_{1}, 0, \ldots\right) \frac{\gamma^{\nu} p_{1 \nu}+m}{p_{1}^{2}-m^{2}}\left(\gamma^{\mu} p_{2 \mu}+m\right)\right]\right\}\left.\frac{-\gamma^{\nu} p_{2 \nu}+m}{p_{2}^{2}-m^{2}}\right|_{p_{1}+p_{2}+p_{3}=0} .
$$

The equality $(3.31)=(3.32)$ is precisely the identity (2.9). The reduced Cg-identities (2.5) and (2.9) imply the perturbative version of the Slavnov-Taylor identity (3.30).

(c) Four-Legs Slavnov-Taylor Identities

We define the connected 4-point Green's functions

$$
\begin{aligned}
& \left.\tilde{L}_{a b c d}^{\alpha \mu \nu \tau}(p, q, k, r)\right|_{p+q+k+r=0}: \stackrel{\text { def }}{=}(2 \pi)^{-6} \int d^{4} x \int d^{4} y \int d^{4} z e^{i p x} e^{i q y} e^{i k z} \\
& <\Omega\left|T\left(A_{\operatorname{int} a}^{\alpha}(x) A_{\operatorname{int} b}^{\mu}(y) A_{\operatorname{int} c}^{\nu}(z) A_{\operatorname{int} d}^{\tau}(0)\right)\right| \Omega>_{c}, \\
& \left.\tilde{H}_{a b c d}^{\lambda \mu \nu}(p, q, k, r) q_{\lambda}\right|_{p+q+k+r=0}: \stackrel{\text { def }}{=}(2 \pi)^{-6} \int d^{4} x \int d^{4} y \int d^{4} z e^{i p x} e^{i q y} e^{i k z} \\
& <\Omega\left|T\left(\tilde{u}_{\operatorname{int} a}(x) u_{\operatorname{int} b}(y) A_{\operatorname{int} c}^{\mu}(z) A_{\operatorname{int} d}^{\nu}(0)\right)\right| \Omega>_{c}, \\
& \left.\tilde{M}_{\alpha \beta a b}^{\mu \nu}(p, q, k, r)\right|_{p+q+k+r=0}: \stackrel{\text { def }}{=}(2 \pi)^{-6} \int d^{4} x \int d^{4} y \int d^{4} z e^{i p x} e^{i q y} e^{i k z} \\
& <\Omega\left|T\left(\psi_{\operatorname{int} \alpha}(x) \bar{\psi}_{\operatorname{int} \beta}(y) A_{\operatorname{int} a}^{\mu}(z) A_{\operatorname{int} b}^{\nu}(0)\right)\right| \Omega>_{c}, \\
& \left.\tilde{F}_{\alpha \beta a b}^{\lambda}(p, q, k, r) r_{\lambda}\right|_{p+q+k+r=0}: \stackrel{\text { def }}{=}(2 \pi)^{-6} \int d^{4} x \int d^{4} y \int d^{4} z e^{i p x} e^{i q y} e^{i k z} \\
& <\Omega\left|T\left(\psi_{\operatorname{int} \alpha}(x) \bar{\psi}_{\operatorname{int} \beta}(y) \tilde{u}_{\operatorname{int} a}(z) u_{\operatorname{int} b}(0)\right)\right| \Omega>_{c}, \\
& \left.S^{F}(p) \gamma^{\mu} \tilde{M}_{\alpha \beta a b}^{1 \nu}(p, q, k, r)\right|_{p+q+k+r=0}: \stackrel{\text { def }}{=} \frac{-g}{2(2 \pi)^{6}} \int d^{4} x \int d^{4} y \int d^{4} z e^{i p x} e^{i q y} e^{i k z} \int d^{4} x_{1} \\
& \left.<\Omega \mid T\left(S^{F}\left(x-x_{1}\right)\left(\lambda_{a^{\prime}}\right)_{\alpha \alpha^{\prime}} \gamma^{\mu} \psi_{\operatorname{int} \alpha^{\prime}}\left(x_{1}\right) u_{\operatorname{int} a^{\prime}}\left(x_{1}\right) \bar{\psi}_{\operatorname{int} \beta}(y) \tilde{u}_{\operatorname{int} a}(z)\right) A_{\operatorname{int} b}^{\nu}(0)\right) \mid \Omega>_{c}, \\
& \left.\tilde{M}_{\alpha \beta a b}^{2 \nu}(p, q, k, r) \gamma^{\mu} S^{F}(-q)\right|_{p+q+k+r=0}: \stackrel{\text { def }}{=} \frac{-g}{2(2 \pi)^{6}} \int d^{4} x \int d^{4} y \int d^{4} z e^{i p x} e^{i q y} e^{i k z} \int d^{4} x_{1} \\
& <\Omega\left|T\left(\psi_{\operatorname{int} \alpha}(x) \bar{\psi}_{\operatorname{int} \beta^{\prime}}\left(x_{1}\right)\left(\lambda_{a^{\prime}}\right)_{\beta^{\prime} \beta} \gamma^{\mu} S^{F}\left(x_{1}-y\right) u_{\operatorname{int} a^{\prime}}\left(x_{1}\right) \tilde{u}_{\operatorname{int} a}(z) A_{\operatorname{int} b}^{\nu}(0)\right)\right| \Omega>_{c} .
\end{aligned}
$$

The latter two Green's functions have one Q-vertex at $x_{1}$, all other vertices are ordinary vertices. In (3.34), (3.36) a factor $q_{\lambda}$ (rsp. $r_{\lambda}$ ) is separated. Similarly to (3.9), (3.16), this factor comes from $\partial_{\lambda} \tilde{u}$ which is contracted with the external vertex $u_{b}(y)\left(\operatorname{rsp} . u_{b}(0)\right)$ in $<\Omega\left|T\left(\ldots u_{\text {int } b}(\ldots) \ldots\right)\right| \Omega>_{c}$. Although 't Hooft [12] and Taylor [10] consider only the case with some of the external momenta on the mass-shell, it is clear from their works how to translate the three-legs Slavnov-Taylor identities (3.23), (3.30) to the four-legs case: Assuming all external momenta $p_{i}(i \in\{1,2,3,4\})$ and all $\left(p_{i}+p_{j}\right)(1 \leq i<j \leq 4)$ to be off-shell, one has

$$
\begin{gathered}
\left.p_{1 \alpha} \tilde{L}_{a b c d}^{\alpha \mu \nu \tau}\left(p_{1}, p_{2}, p_{3}, p_{4}\right)\right|_{p_{1}+p_{2}+p_{3}+p_{4}=0}=\tilde{H}_{a b c d}^{\lambda \nu \tau}\left(p_{1}, p_{2}, p_{3}, p_{4}\right)\left(g_{\lambda}^{\mu} p_{2}^{2}-p_{2}^{\mu} p_{2 \lambda}\right)+ \\
+\tilde{H}_{a c d b}^{\lambda \tau \mu}\left(p_{1}, p_{3}, p_{4}, p_{2}\right)\left(g_{\lambda}^{\nu} p_{3}^{2}-p_{3}^{\nu} p_{3 \lambda}\right)+\left.\tilde{H}_{a d b c}^{\lambda \mu \nu}\left(p_{1}, p_{4}, p_{2}, p_{3}\right)\left(g_{\lambda}^{\tau} p_{4}^{2}-p_{4}^{\tau} p_{4 \lambda}\right)\right|_{p_{1}+p_{2}+p_{3}+p_{4}=0}, \\
-\left.p_{3 \mu} \tilde{M}_{\alpha \beta a b}^{\mu \nu}\left(p_{1}, p_{2}, p_{3}, p_{4}\right)\right|_{p_{1}+p_{2}+p_{3}+p_{4}=0}=S^{F}\left(p_{1}\right)\left(\gamma^{\mu} p_{1 \mu}-m\right) \tilde{M}_{\alpha \beta a b}^{1 \nu}\left(p_{1}, p_{2}, p_{3}, p_{4}\right)+
\end{gathered}
$$




$$
\begin{gathered}
+\tilde{M}_{\alpha \beta a b}^{2 \nu}\left(p_{1}, p_{2}, p_{3}, p_{4}\right)\left(\gamma^{\mu} p_{2 \mu}+m\right) S^{F}\left(-p_{2}\right)-\left.\tilde{F}_{\alpha \beta a b}^{\lambda}\left(p_{1}, p_{2}, p_{3}, p_{4}\right)\left(g_{\lambda}^{\nu} p_{4}^{2}-p_{4}^{\nu} p_{4 \lambda}\right)\right|_{p_{1}+\ldots=0} \equiv \\
\equiv(2 \pi)^{-2}\left[\tilde{M}_{\alpha \beta a b}^{1 \nu}\left(p_{1}, p_{2}, p_{3}, p_{4}\right)-\tilde{M}_{\alpha \beta a b}^{2 \nu}\left(p_{1}, p_{2}, p_{3}, p_{4}\right)\right]- \\
\quad-\left.\tilde{F}_{\alpha \beta a b}^{\lambda}\left(p_{1}, p_{2}, p_{3}, p_{4}\right)\left(g_{\lambda}^{\nu} p_{4}^{2}-p_{4}^{\nu} p_{4 \lambda}\right)\right|_{p_{1}+p_{2}+p_{3}+p_{4}=0}
\end{gathered}
$$

and these are all Slavnov-Taylor identities with four external legs. The identity (3.39) in terms of one-particle irreducible Green's functions can be found in [19], formula (1.2).

We claim that the perturbative versions of the Slavnov-Taylor identities (3.39-40) can be proven from our reduced two-, three- and four-legs Cg-identities. Since the proof of this statement is a straight-forward calculation, completely analogous to the three-legs case, but very much longer, we only describe the procedure here. First one has to express the GellMann Low series of the connected Green's functions (3.33-38) in terms of our $t$-distributions. Besides the non-degenerate terms $\sim \tilde{t}_{B_{1} B_{2} B_{3} B_{4}}^{(n)}$ of order $n$ there are degenerate ones, namely three-legs distributions in order $(n-1)$ with one separated vertex (fig.3a), and two-legs distributions in order $(n-2)$ with two separated vertices (figs.3b,c). In the case of $\tilde{L}$ (3.33) one should not forget the two-legs distribution $\tilde{t}_{A A}^{(n-2)}$ combined with a four-gluon vertex (fig.3d). All other terms with four-gluon vertices (e.g. fig.1) are contained in the $\tilde{t}$ distributions. Next we insert these $n$-th order expressions for the connected Green's functions into (3.39-40). In order to obtain agreement of the l.h. and r.h. sides in the resulting equations one uses

-the reduced Cg-identities (2.24), (2.14), (2.5), the covariant decomposition (2.4) of $t_{u \tilde{u}}$ and the Jacobi-identity of the structure constants $f_{a b c}$ in the case of (3.39);

-and the reduced Cg-identities (2.23), (2.9), (2.14), (2.5), again (2.4) and the identity $\left[\lambda_{a}, \lambda_{b}\right]=2 i f_{a b c} \lambda_{c}$ in the case of (3.40).

\section{(d) Concluding Remarks}

There are also Cg- and Slavnov-Taylor identities with five or more external legs. The crucial step in the proof of gauge invariance is the distribution splitting. In this process the mentioned $\mathrm{Cg}$-identities cannot be violated $[4,5]$. Consequently, they give no further restriction on the normalization of the $t$-distributions (sect.2d). Therefore, they are not of great interest and we do not consider them here.

The original Cg-identities $[2,3,5]$ contain more information than the Slavnov-Taylor identities, because their coordinates refer to external and to inner vertices, whereas in the Green's functions the inner vertices are integrated out with $g(x) \equiv 1$. In momentum space, this integration corresponds to the limit of vanishing inner momenta and vanishing $p_{n} \stackrel{\text { def }}{=}-$ $\left(p_{1}+p_{2}+\ldots+p_{n-1}\right)$. In sect.2 we have seen that the unphysical Q-vertex can only be eliminated from the Cg-identities by taking this limit. The result of this elimination are seven reduced Cg-identities. Let us first consider only five of them: (2.5) with two legs, (2.9) and (2.14) with three legs, and (2.23-24) with four legs. We have proven that these five identities imply the perturbative versions of all Slavnov-Taylor identities up to four external legs, namely (3.4) with two legs, (3.23) and (3.30) with three legs, and (3.3940) with four legs. However, our calculations yield more information: In the framework of perturbation theory these five Slavnov-Taylor identities are equivalent to the above five reduced Cg-identities.

We turn to the remaining two reduced Cg-identities: (2.15) with three legs and (2.25) with four legs. Most probably, they can be expressed by two identities between connected Green's functions. The connected Green's function with non-degenerate part $\sim \bar{t}_{u u \tilde{u}}^{3}$ (rsp. 
$\left.\sim \tilde{t}_{u u \tilde{u} A}^{3 \nu}\right)$, the latter appears in (2.15) (rsp. (2.25)), has one Q-vertex $T_{1 / 1}^{u}(1.11)$, which is directly contracted with the external vertex $u(z)$ in $<\Omega\left|T\left(\tilde{u}_{\text {int }}(x) \tilde{u}_{\text {int }}(y) u_{\text {int }}(z)\left[A_{\text {int }}(0)\right]\right)\right|$ $\Omega>_{c}$. This is analogous to $\tilde{\Gamma}^{1 / 2}(3.26-27)$ or $\tilde{M}^{1 / 2}(3.37-38)$. However, the reduced $C g$ identities (2.15), (2.25) give no restriction on the normalization of the physical theory, in contrast to (2.9), (2.23), which contain distributions with one Q-vertex, too (sect.2d). Therefore, we do not consider the identities of Green's functions corresponding to (2.15), (2.25). They cannot be found in the literature either.

\section{Appendix: Epstein and Glaser's definition of Green's functions}

Following Epstein and Glaser (sect.8.1 of [6]) we define a bigger theory by giving its first order

$$
S_{1}\left(g, g_{1}, \ldots g_{l}\right) \stackrel{\text { def }}{=} \int d^{4} x\left\{T_{1}(x) g(x)+i \Phi_{1}(x) g_{1}(x)+\ldots+i \Phi_{l}(x) g_{l}(x)\right\} .
$$

The interacting field $\Phi_{j \text { int }}(x ; g)$ is defined by $[20,6,21]$

$$
\left.\Phi_{j \text { int }}(x ; g) \stackrel{\text { def }}{=} S^{-1}(g, 0, \ldots 0) \frac{\delta S\left(g, g_{1}, \ldots g_{l}\right)}{i \delta g_{j}(x)}\right|_{g_{1}=\ldots g_{l}=0}, \quad j \in\{1, \ldots l\} .
$$

Higher functional derivatives

$$
\left.\hat{T}_{j_{1} \ldots j_{r}}\left(x_{1}, \ldots x_{r} ; g\right) \stackrel{\text { def }}{=} S^{-1}(g, 0, \ldots 0) \frac{\delta^{r} S\left(g, g_{1}, \ldots g_{l}\right)}{i^{r} \delta g_{j_{1}}\left(x_{1}\right) \ldots \delta g_{j r}\left(x_{r}\right)}\right|_{g_{1}=\ldots g_{l}=0},
$$

$j_{1}, \ldots j_{r} \in\{1, \ldots l\}$, define time-ordered products of these interacting fields [6]

$$
\hat{T}_{j_{1} \ldots j r}\left(x_{1}, \ldots x_{r} ; g\right)=\Phi_{j_{\pi 1} \text { int }}\left(x_{\pi 1} ; g\right) \Phi_{j_{\pi 2} \text { int }}\left(x_{\pi 2} ; g\right) \ldots \Phi_{j \pi r \text { int }}\left(x_{\pi r} ; g\right),
$$

where $\pi \in \mathcal{S}_{r}$ is a permutation which puts the coordinates in time-order $x_{\pi 1} \succeq x_{\pi 2} \succeq \ldots x_{\pi r}$ $\left(x \succeq y\right.$ means $\left.x \in \mathbf{R}^{4} \backslash\left(y+\bar{V}^{-}\right)\right)$. One easily obtains the perturbative expansion of (A.3)

$$
\hat{T}_{j_{1} \ldots j_{r}}\left(x_{1}, \ldots x_{r} ; g\right)=\sum_{n=0}^{\infty} \frac{1}{i^{r} n !} \int d^{4} y_{1} \ldots d^{4} y_{n} A_{0 \ldots 0 j_{1} \ldots j_{r}}\left(y_{1}, \ldots y_{n} ; x_{1}, \ldots x_{r}\right) g\left(y_{1}\right) \ldots g\left(y_{n}\right),
$$

with

$$
A_{0 \ldots 0 j_{1} \ldots j_{r}}\left(y_{1}, \ldots y_{n} ; x_{1}, \ldots x_{r}\right) \stackrel{\text { def }}{=} \sum_{I \subset Y} \bar{T}_{0 \ldots 0}(I) T_{0 \ldots 0 j_{1} \ldots j_{r}}\left(Y \backslash I ; x_{1}, \ldots x_{r}\right),
$$

where $Y \stackrel{\text { def }}{=}\left\{y_{1}, \ldots y_{n}\right\}$ and a lower index 0 means that the vertex at the corresponding position in the argument is an ordinary vertex $T_{1}(x)$, an index $j$ indicates a vertex $i \Phi_{j}(x)$. By means of the causal factorization of $\bar{T}_{0 \ldots 0}(I)$ and $T_{0 \ldots 0 j_{1} \ldots j_{r}}\left(Y \backslash I ; x_{1}, \ldots x_{r}\right)$ one easily proves $\operatorname{supp} A_{0 \ldots 0 j_{1} \ldots j_{r}}\left(y_{1}, \ldots y_{n} ; x_{1}, \ldots x_{r}\right) \subset\left\{\left(y_{1}, \ldots y_{n} ; x_{1}, \ldots x_{r}\right) \mid y_{i} \in\left\{x_{1}, \ldots x_{r}\right\}+\bar{V}^{-}, \forall i=1, \ldots n\right\}$. 
The time-ordering (A.4) relies on this support property (see [6]). The r-point Green's function (corresponding to (3.1)) is defined [6] by the vacuum expectation value of (A.3)

$$
\begin{gathered}
<0\left|\hat{T}_{j_{1} \ldots j_{r}}\left(x_{1}, \ldots x_{r} ; g\right)\right| 0>= \\
=\sum_{n=0}^{\infty} \frac{1}{i^{r} n !} \int d^{4} y_{1} \ldots d^{4} y_{n}<0\left|A_{0 \ldots 0 j_{1} \ldots j_{r}}\left(y_{1}, \ldots y_{n} ; x_{1}, \ldots x_{r}\right)\right| 0>g\left(y_{1}\right) \ldots g\left(y_{n}\right)
\end{gathered}
$$

in the Fock vacuum $\mid 0>$ of free fields. This is in contrast to (3.1), where the interacting vacuum $\mid \Omega>$ is used. However, (A.5) is an expansion of $T\left(\Phi_{j_{1} \text { int }}\left(x_{1}\right) \ldots \Phi_{j_{r} \text { int }}\left(x_{r}\right)\right)$ in terms of free fields, it is an operator in the Fock space of free fields, consequently the free vacuum must be used.

The adiabatic switching with $g\left(y_{1}\right) \ldots g\left(y_{n}\right), g \in \mathcal{S}\left(\mathbf{R}^{4}\right)$ in (A.8) is an infrared regularization, which should finally be removed. The crucial question is whether the adiabatic limit $g \rightarrow 1$ of (A.8) exists in the sense of tempered distributions in $\left(x_{1}, \ldots x_{r}\right) \in \mathbf{R}^{4 r}$, if a suitable normalization is chosen (see (3.3a) and the remark at the end of this appendix). We shall meet an explicit example where this limit exists, although the GellMann Low expression does not. For massive theories Epstein and Glaser (sect.8.2 of [6]) prove the existence of the adiabatic limit of (A.8). Moreover they show that this limit possesses all the linear properties of a Green's function such as translation invariance, Lorentz covariance, causality and the spectral condition. Blanchard and Seneor [22] prove similar results for theories with (some) massless particles such as QED and $\lambda: \Phi^{2 n}$ : theories. However, in our Yang-Mills model with matter fields the infrared behaviour is worse ([23] or compare (3.3a) and (3.3b)) and I do not know whether the adiabatic limit of (A.8) exists. But our assumption (1.24-25) (extended to the retarded $\hat{r}$ - and advanced $\hat{a}$-distributions $[6,8]$ ) implies its existence, if all (sums of) external momenta are off-shell. This relies on the fact that the overlapping singularities (appearing in the products of propagators and non-degenerate $\hat{t}, \hat{r}, \hat{a}$-distributions) are excluded in this region.

In order to compare the definition (A.8) of Green's functions with the usual definition (3.1), we compute (A.8) for the gluon propagator in lowest non-trivial order: Choosing

$$
\Phi_{1}(x) g_{1}(x)=A_{a \mu}(x) g_{1 a}^{\mu}(x)
$$

in (A.1), one obtains

$$
\begin{gathered}
<0\left|A_{0011}\left(y_{1}, y_{2} ; x_{1}, x_{2}\right)^{\mu \nu}\right| 0>=C\left[\tilde{t}_{A A}^{\mu \nu}\left(y_{1}-y_{2}\right) D_{0}^{\mathrm{av}}\left(y_{1}-x_{1}\right) D_{0}^{\mathrm{av}}\left(y_{2}-x_{2}\right)+\right. \\
\left.+\tilde{a}_{A A}^{\mu \nu}\left(y_{1}-y_{2}\right) D_{0}^{+}\left(y_{1}-x_{1}\right) D_{0}^{\mathrm{av}}\left(y_{2}-x_{2}\right)+\tilde{r}_{A A}^{\mu \nu}\left(y_{1}-y_{2}\right) D_{0}^{\mathrm{av}}\left(y_{1}-x_{1}\right) D_{0}^{+}\left(y_{2}-x_{2}\right)\right]+C\left[y_{1} \leftrightarrow y_{2}\right],
\end{gathered}
$$

where $\tilde{r}_{A A}\left(\tilde{a}_{A A}\right)$ are the retarded (advanced) distributions [8] belonging to $\tilde{t}_{A A}(2.1)$ and

$D_{m}^{\text {av }}(x) \stackrel{\text { def }}{=}-(2 \pi)^{-4} \int d^{4} k \frac{e^{-i k x}}{k^{2}-m^{2}-i k_{0} 0}, \quad D_{m}^{ \pm}(x) \stackrel{\text { def }}{=} \frac{i}{(2 \pi)^{3}} \int d^{4} k e^{-i k x} \delta\left(k^{2}-m^{2}\right) \Theta\left( \pm k_{0}\right)$.

(Note $m=0$ in (A.10).) $\mathrm{C}$ is a constant factor which is not of interest here. Obviously, (A.10) has the support property (A.7) and it does not agree with the corresponding expression

$$
C \tilde{t}_{A A}^{\mu \nu}\left(y_{1}-y_{2}\right) D_{0}^{F}\left(y_{1}-x_{1}\right) D_{0}^{F}\left(y_{2}-x_{2}\right)+\left(y_{1} \leftrightarrow y_{2}\right)
$$

$\left(D_{m}^{F}\right.$ is the Feynman propagator, $D_{m}^{F}=D_{m}^{\text {av }}+D_{m}^{+}$) in the Gell-Mann Low series (without integrating over $y_{1}, y_{2}$, compare (3.2-3)). Let us study the adiabatic limit of (A.10) inserted 
into (A.8) in momentum space. We do this by replacing $g(x)$ by $g_{\epsilon}(x)(1.27)$ and considering $\epsilon \rightarrow 0, \epsilon>0$. Then, with (1.28) we obtain

$$
\begin{gathered}
\lim _{\epsilon \rightarrow 0} \int d^{4} x_{1} d^{4} x_{2} e^{i\left(p_{1} x_{1}+p_{2} x_{2}\right)} \int d^{4} y_{1} d^{4} y_{2}<0\left|A_{0011}\left(y_{1}, y_{2} ; x_{1}, x_{2}\right)\right| 0>g_{\epsilon}\left(y_{1}\right) g_{\epsilon}\left(y_{2}\right) \sim \\
\sim \lim _{\epsilon \rightarrow 0} \delta^{4}\left(p_{1}+p_{2}\right) \int d^{4} k_{1} d^{4} k_{2} \hat{g}_{0}\left(k_{1}\right) \hat{g}_{0}\left(k_{2}\right)\left[\tilde{t}_{A A}\left(p_{1}-\epsilon k_{1}\right) \hat{D}_{m}^{\mathrm{av}}\left(-p_{1}\right) \hat{D}_{m}^{\mathrm{av}}\left(p_{1}-\epsilon\left(k_{1}+k_{2}\right)\right)+\right. \\
\left.+\tilde{a}_{A A}\left(p_{1}-\epsilon k_{1}\right) \hat{D}_{m}^{+}\left(-p_{1}\right) \hat{D}_{m}^{\mathrm{av}}\left(p_{1}-\epsilon\left(k_{1}+k_{2}\right)\right)+\tilde{r}_{A A}\left(p_{1}-\epsilon k_{1}\right) \hat{D}_{m}^{\mathrm{av}}\left(-p_{1}\right) \hat{D}_{m}^{+}\left(p_{1}-\epsilon\left(k_{1}+k_{2}\right)\right)\right]= \\
=\lim _{\epsilon \rightarrow 0} \delta^{4}\left(p_{1}+p_{2}\right) \int d^{4} k_{1} d^{4} k_{2} \hat{g}_{0}\left(k_{1}\right) \hat{g}_{0}\left(k_{2}\right)\left[\tilde{t}_{A A}\left(p_{1}-\epsilon k_{1}\right) \hat{D}_{m}^{F}\left(p_{1}\right) \hat{D}_{m}^{F}\left(p_{1}-\epsilon\left(k_{1}+k_{2}\right)\right)-\right. \\
\quad+\left(\tilde{t}_{A A}\left(p_{1}-\epsilon k_{1}\right)+\tilde{a}_{A A}^{\prime}\left(p_{1}-\epsilon k_{1}\right)+\tilde{r}_{A A}^{\prime}\left(p_{1}-\epsilon k_{1}\right)\right) \hat{D}_{m}^{-}\left(p_{1}\right) \hat{D}_{m}^{+}\left(p_{1}-\epsilon\left(k_{1}+k_{2}\right)\right)+ \\
\left.-\tilde{a}_{A A}^{\prime}\left(p_{1}-\epsilon k_{1}\right) \hat{D}_{m}^{-}\left(p_{1}\right) \hat{D}_{m}^{F}\left(p_{1}-\epsilon\left(k_{1}+k_{2}\right)\right)+\tilde{r}_{A A}^{\prime}\left(p_{1}-\epsilon k_{1}\right) \hat{D}_{m}^{F}\left(p_{1}\right) \hat{D}_{m}^{+}\left(p_{1}-\epsilon\left(k_{1}+k_{2}\right)\right)\right],
\end{gathered}
$$

where $m=0 . \quad \tilde{a}_{A A}^{\prime}=\tilde{a}_{A A}-\tilde{t}_{A A}$ and $\tilde{r}_{A A}^{\prime}=\tilde{r}_{A A}-\tilde{t}_{A A}$ are the usual $a^{\prime}, r^{\prime}$-distributions, which have the factors $[2]$

$$
\tilde{a}_{A A}^{\prime}(k) \sim \Theta\left(k^{2}-m_{1}^{2}\right) \Theta\left(k^{0}\right), \quad \tilde{r}_{A A}^{\prime}(k) \sim \Theta\left(k^{2}-m_{1}^{2}\right) \Theta\left(-k^{0}\right) .
$$

(More precisely, $\tilde{a}_{A A}^{\prime}, \tilde{r}_{A A}^{\prime}$ are the sum of the gluon, ghost and matter loops. For the first two we have $m_{1}=0$ and for the latter $m_{1}=2 m_{\psi} \geq 0$.) For simplicity we assume $\hat{g}_{0}$ to have compact support. We first consider a massive theory: $m, m_{1}>0$. Then, the adiabatic limit in (A.13) exists in the sense of tempered distributions in $\left(p_{1}, p_{2}\right) \in \mathbf{R}^{8}$ [6]. Due to the support properties of $\hat{D}_{m}^{+}$(A.11) and $\tilde{a}_{A A}^{\prime}, \tilde{r}_{A A}^{\prime}$ (A.14) the terms in (A.13b) vanish for $\epsilon>0$ sufficently small. The remaining term (A.13a) is an infrared-improved version of the Gell-Mann Low expression (3.3). We now turn to our model: In order to avoid discussions about infrared divergences, we only consider the region $p_{1}^{2} \neq 0$. There, the adiabatic limit of (A.13a,b) and the Gell-Mann Low expression exist. Moreover, for $p_{1}$ off-shell, the terms (A.13b) vanish if $\epsilon>0$ is sufficently small and the two definitions of the Gluon propagator agree in second order. This holds true also for the ghost and matter propagators (compare (3.5-6)) since they have a similar structure (A.10), (A.12), (A.13).

In order to get a better understanding of the adiabatic limit of (A.8), we consider the electron propagator in second order in QED with the mass of the electron $m>0$. The Gell-Mann Low expression (see (3.8))

$$
i S^{(2)}(p)=-\frac{\gamma^{\nu} p_{\nu}+m}{p^{2}-m^{2}+i 0} t_{\bar{\psi} \psi}^{(2)}(p) \frac{\gamma^{\nu} p_{\nu}+m}{p^{2}-m^{2}+i 0}
$$

does not exist for $p^{2} \approx m^{2}$, because in this region $t_{\bar{\psi} \psi}^{(2)}[8]$ has the behaviour

$$
t_{\bar{\psi} \psi}^{(2)}(p) \sim\left\{\log \left(1-\frac{p^{2}+i 0}{m^{2}}\right)\left(m-\frac{\gamma^{\nu} p_{\nu}}{2}\right)\left(1-\frac{m^{2}}{p^{2}}\right)+C\left(\gamma^{\nu} p_{\nu}-m\right)\right\}
$$

where $C$ is an arbitrary constant and the mass normalization $t \frac{(2)}{\psi} \psi\left(\gamma^{\nu} p_{\nu}=m\right)=0$ is done. The Epstein-Glaser expression is obtained from (A.13a,b) by replacing $\hat{D}_{m}^{b}(p)(b= \pm, F)$ by $\hat{S}_{m}^{b}(p) \stackrel{\text { def }}{=} \pm\left(\gamma^{\nu} p_{\nu}+m\right) \hat{D}_{m}^{b}(p)$ and $\tilde{f}_{A A}(p)\left(f=t, a^{\prime}, r^{\prime}\right)$ by $f_{\bar{\psi} \psi}(p)$. Note that $\hat{S}_{m}^{ \pm}(p)$, 
rsp. $a_{\bar{\psi} \psi}^{\prime}(k), r_{\bar{\psi} \psi}^{\prime}(k)$ have the same support properties as $\hat{D}_{m}^{ \pm}(p)(\mathrm{A} .11)$, rsp. $\tilde{a}_{A A}^{\prime}(k), \tilde{r}_{A A}^{\prime}(k)$ (A.14) (the latter with $m_{1}$ replaced by $m$ ). Consequently, the terms corresponding to (A.13b) vanish in the adiabatic limit and it remains

$$
\begin{gathered}
\sim \lim _{\epsilon \rightarrow 0} \delta^{4}\left(p_{1}+p_{2}\right) \int d^{4} k_{1} d^{4} k_{2} \hat{g}_{0}\left(k_{1}\right) \hat{g}_{0}\left(k_{2}\right) \frac{\gamma^{\nu} p_{1 \nu}+m}{p_{1}^{2}-m^{2}+i 0} . \\
\cdot t \frac{(2)}{\psi}\left(p_{1}-\epsilon k_{1}\right) \frac{\gamma^{\nu}\left(p_{1}-\epsilon\left(k_{1}+k_{2}\right)\right)_{\nu}+m}{\left(p_{1}-\epsilon\left(k_{1}+k_{2}\right)\right)^{2}-m^{2}+i 0} .
\end{gathered}
$$

An explicit calculation shows that this adiabatic limit exists in the sense of tempered distributions in $\left(p_{1}, p_{2}\right) \in \mathbf{R}^{8}$ : The critical term is the product of $f(p) \stackrel{\text { def }}{=} \frac{1}{p^{2}-m^{2}+i 0}=P\left(\frac{1}{p^{2}-m^{2}}\right)-$ $i \pi \delta\left(p^{2}-m^{2}\right)$ with $h\left(p-\epsilon k_{1}\right) \stackrel{\text { def }}{=} \log \left(1-\frac{\left(p-\epsilon k_{1}\right)^{2}+i 0}{m^{2}}\right)=\log \left|1-\frac{\left(p-\epsilon k_{1}\right)^{2}}{m^{2}}\right|-i \pi \Theta\left(\left(p-\epsilon k_{1}\right)^{2}-m^{2}\right)$. Smeared out in $p$ with an arbitrary test-function, the products $(\operatorname{Re} f)(\operatorname{Re} h)$ and $(\operatorname{Im} f)(\operatorname{Im} h)$ are finite for $\epsilon \rightarrow 0$, whereas $(\operatorname{Re} f)(\operatorname{Im} h)$ and $(\operatorname{Im} f)(\operatorname{Re} h)$ have divergent terms $\sim \log |\epsilon|$. But the latter cancel exactly. This is in accordance with the general result of Blanchard and Seneor [22]. Obviously, the Epstein-Glaser expression (A.15b) is an infrared-improved version of (A.15). The existence of Green's functions in perturbation theory does not only depend on the behaviour of the specific theory near the mass-shell (e.g. (3.3a,b), (A.15)), it depends also on the definition of the product of distributions with overlapping singularities. In the following we always assume the Gell-Mann Low expressions to be infrared-improved in the sense of (A.15b).

In the massive case we now generalize the above reasoning in (A.13).

Proposition: The adiabatic limit of Epstein and Glaser's Green's function (A.8) (which exists if the normalization constants are chosen appropriately) agrees with the infrared-improved Gell-Mann Low series in all orders.

Proof: Let us consider the expansion of the terms on the r.h.s. of (A.6) in normally ordered form. The terms with vacuum subdiagram(s) must cancel. This is due to the support property (A.7) of $A_{0 \ldots 0 j_{1} \ldots j_{r}}$. Therefore, we may omit the terms with vacuum subdiagram(s) on the r.h.s. of (A.6). This omission will be denoted by a lower index 0 in $<0|\ldots| 0>_{0}$. (In the remark below we shall see that the adiabatic limit of an arbitrary vacuum diagram vanishes and, therefore, this holds true also for the terms with vacuum subdiagram(s). However, this information is not needed in the proof here.)

The infrared-improved Gell-Mann Low expression in n-th order is obtained by the adiabatic limit of the $I=\emptyset$ term in (A.6)

$$
\lim _{\epsilon \rightarrow 0} \frac{1}{i^{r} n !} \int d^{4} y_{1} \ldots d^{4} y_{n}<0\left|T_{0 \ldots 0 j_{1} \ldots j_{r}}\left(y_{1}, \ldots y_{n} ; x_{1}, \ldots x_{r}\right)\right| 0>_{0} g_{\epsilon}\left(y_{1}\right) \ldots g_{\epsilon}\left(y_{n}\right)
$$

with the above mentioned omission. Consequently, we merely have to prove that the $I \neq \emptyset$ terms in (A.6) vanish in the adiabatic limit,

$$
\lim _{\epsilon \rightarrow 0} \int d^{4} y_{1} \ldots d^{4} y_{n}<0\left|\bar{T}_{0 \ldots 0}(I) T_{0 \ldots 0 j_{1} \ldots j_{r}}\left(Y \backslash I ; x_{1}, \ldots x_{r}\right)\right| 0>_{0} g_{\epsilon}\left(y_{1}\right) \ldots g_{\epsilon}\left(y_{n}\right)=0
$$

for $I \neq \emptyset$. We do this by considering an arbitrary term belonging to $<0 \mid \bar{T}_{0 \ldots 0}(I)$ $T_{0 \ldots 0 j_{1} \ldots j_{r}}\left(Y \backslash I ; x_{1}, \ldots x_{r}\right) \mid 0>_{0}$. It has the form

$$
t_{1}\left(y_{1}-y_{s}, \ldots y_{s-1}-y_{s}\right) P_{1}^{+}\left(y_{j_{1}}-z_{i_{1}}\right) \ldots
$$




$$
\ldots P_{l}^{+}\left(y_{j_{l}}-z_{i}\right) t_{2}\left(y_{s+1}-x_{r}, \ldots y_{n}-x_{r} ; x_{1}-x_{r}, \ldots x_{r-1}-x_{r}\right)
$$

where $\left\{y_{1}, \ldots y_{s}\right\}=I, \quad\left\{y_{s+1}, \ldots y_{n}\right\}=Y \backslash I, y_{j_{1}}, \ldots y_{j_{l}} \in I, z_{i_{1}}, \ldots z_{i_{l}} \in(Y \backslash I) \cup\left\{x_{1}, \ldots x_{r}\right\}$, and

$$
P_{j}^{+}(x)=D_{m_{j}}^{+}(x), \partial D_{m_{j}}^{+}(x), \partial \partial D_{m_{j}}^{+}(x), \ldots S_{m_{j}}^{+}(x), S_{m_{j}}^{-}(-x), \quad m_{j}>0, \forall y
$$

$\left(S_{m_{j}}^{ \pm}\right.$means a contraction of fermionic matter fields $\bar{\psi}$ and $\psi$.) Note that $y_{j_{m}}=y_{j_{s}}$ or $z_{i m}=z_{i_{s}}$ is possible in (A.18) for $m \neq s$. We perform the Fourier transformation in the relative coordinates $\tilde{y}_{i} \stackrel{\text { def }}{=} y_{i}-x_{r}(i=1, \ldots, n)$ and $\tilde{x}_{j} \stackrel{\text { def }}{=} x_{j}-x_{r}(j=1, \ldots, r-1)$

$$
\begin{gathered}
\int d^{4} \tilde{y}_{1} \ldots d^{4} \tilde{y}_{n} e^{i\left(k_{1} \tilde{y}_{1}+\ldots+k_{n} \tilde{y} n\right)} \int d^{4} \tilde{x}_{1} \ldots d^{4} \tilde{x}_{r-1} e^{i\left(p_{1} \tilde{x}_{1}+\ldots+p_{r-1} \tilde{x}_{r-1}\right)}(A .18) \sim \\
\sim \int d^{4} q_{1} \ldots d^{4} q_{l-1} \hat{t}_{1}\left(\ldots\left(k_{i}, p_{j}, q_{m}\right) \ldots\right) \hat{P}_{1}^{+}\left(q_{1}\right) \ldots \\
\ldots \hat{P}_{l-1}^{+}\left(q_{l-1}\right) \hat{P}_{l}^{+}\left(-k_{1}-k_{2}-\ldots-k_{s}-q_{1}-\ldots-q_{l-1}\right) \hat{t}_{2}\left(\ldots\left(k_{i}, p_{j}, q_{m}\right) \ldots\right) .
\end{gathered}
$$

If $t_{1}$ (or $t_{2}$ ) belongs to a disconnected diagram, $\hat{t}_{1}$ (rsp. $\hat{t}_{2}$ ) contains (a) $\delta^{4}$-distribution(s) and the number of loop integrations in (A.20) (which seems to be $(l-1)$ ) is reduced. By means of (1.27) the adiabatic limit of (A.18) (rsp. (A.20)) can be written in the following form in momentum space

$$
\begin{array}{r}
\sim \lim _{\epsilon \rightarrow 0} \int d^{4} k_{1} \ldots d^{4} k_{n} \hat{g}_{0}\left(k_{1}\right) \ldots \hat{g}_{0}\left(k_{n}\right) \int d^{4} q_{1} \ldots d^{4} q_{l-1} \hat{t}_{1}\left(\ldots\left(\epsilon k_{i}, p_{j}, q_{m}\right) \ldots\right) \\
\hat{P}_{1}^{+}\left(q_{1}\right) \ldots \hat{P}_{l-1}^{+}\left(q_{l-1}\right) \hat{P}_{l}^{+}\left(-\epsilon\left(k_{1}+\ldots+k_{s}\right)-q_{1}-\ldots-q_{l-1}\right) \hat{t}_{2}\left(\ldots\left(\epsilon k_{i}, p_{j}, q_{m}\right) \ldots\right) .
\end{array}
$$

Due to

$$
\hat{P}_{j}^{+}(q) \sim \delta\left(q^{2}-m_{j}^{2}\right) \Theta\left(q^{0}\right), \quad \forall j=1, \ldots l
$$

the limit (A.21) vanishes. Usually the case $l=1$ does not appear, because there are no 1-leg (sub)diagrams. But our proof holds true also for $l=1$. If we have no contraction in (A.18) $(l=0)$, the factor $t_{1}$ corresponds to a vacuum subdiagram and, therefore, such a term is omitted in (A.17).

Let us try to adopt this proof to the massless case. Then, the terms with vacuum subdiagram(s) are logarithmic divergent in the adiabatic limit (see (A.31) below). This does not matter since these terms are omitted. Nevertheless the proof does not work: Assuming in (A.21) for simplicity $l=2, P_{1}^{+}, P_{2}^{+}=D_{0}^{+}$and $\hat{t}_{1}, \hat{t}_{2}$ to be independent on $q \equiv q_{1}$, we obtain for the $q$-integration

$$
\int d^{4} q \delta\left(q^{2}\right) \Theta\left(q^{0}\right) \delta\left((\epsilon k-q)^{2}\right) \Theta\left(\epsilon k^{0}-q^{0}\right)=\frac{\pi}{2} \Theta\left(\epsilon^{2} k^{2}\right) \Theta\left(\epsilon k^{0}\right)=\frac{\pi}{2} \Theta\left(k^{2}\right) \Theta\left(k^{0}\right) .
$$

The corresponding result in the massive case $\left(P_{1}^{+}=D_{m_{1}}^{+}, P_{2}^{+}=D_{m_{2}}^{+}\right)$is $\frac{\pi}{2} \Theta\left(\epsilon^{2} k^{2}-\left(m_{1}+\right.\right.$ $\left.\left.m_{2}\right)^{2}\right) \Theta\left(k^{0}\right)$. But in (A.23) $\epsilon$ drops out and, consequently, the above method of proof fails. However, the limit $\epsilon \rightarrow 0$ of (A.23) exists.

Remark about the adiabatic limit of vacuum diagrams: The adiabatic limit can only exist if certain normalization constants are chosen in an appropriate way. This was already noted in (3.3a), (A.15a) and it can be demonstrated in a very simple way for the vacuum 
diagrams. First we consider a massive theory. We do not need the explicit form of the $t$-distributions for the vacuum diagrams in this case. Since the inner momenta go to zero in the adiabatic limit, it suffices to know the existence of the central solution $\hat{t}_{0}\left(p_{1}, \ldots p_{n-1}\right)$ of the distribution splitting $[6,8,18]$

$$
\left(D^{a} \hat{t}_{0}\right)(0, \ldots 0)=0, \quad \forall|a| \leq \omega,
$$

and that the vacuum diagrams have the singular order (1.17)

$$
\omega=4 .
$$

These two assumptions are usually fulfiled in a massive, renormalizable theory in $d=4$ dimensions. The general splitting solution is (see (1.16))

$$
\hat{t}\left(p_{1}, \ldots p_{n-1}\right)=\hat{t}_{0}\left(p_{1}, \ldots p_{n-1}\right)+C_{0}+\sum_{i j} C_{i j} p_{i} p_{j}+\sum_{i j k l} C_{i j k l}\left(p_{i} p_{j}\right)\left(p_{k} p_{l}\right),
$$

where $C_{0}, C_{i j}, C_{i j k l}$ are arbitrary constants. With (1.27) we obtain for the vacuum expectation value of the n-th order S-matrix

$$
\begin{aligned}
& \lim _{\epsilon \rightarrow 0}\left(\Omega, S_{n}\left(g_{\epsilon}\right) \Omega\right)=\frac{1}{n !} \lim _{\epsilon \rightarrow 0} \int d^{4} x_{1} \ldots d^{4} x_{n} g_{\epsilon}\left(x_{1}\right) \ldots g_{\epsilon}\left(x_{n}\right) t\left(x_{1}-x_{n}, \ldots, x_{n-1}-x_{n}\right)= \\
= & (2 \pi)^{2} \lim _{\epsilon \rightarrow 0} \epsilon^{-4} \int d^{4} p_{1} \ldots d^{4} p_{n-1} \hat{g}_{0}\left(-p_{1}\right) \ldots \hat{g}_{0}\left(-p_{n-1}\right) \hat{g}_{0}\left(p_{1}+\ldots+p_{n-1}\right) \hat{t}\left(\epsilon p_{1}, \ldots, \epsilon p_{n-1}\right) .
\end{aligned}
$$

Inserting (A.26) and remembering (A.24) we realize that the adiabatic limit exists if and only if

$$
C_{0}=0, \quad C_{i j}=0 \quad \forall i, j .
$$

Uniqueness of the adiabatic limit, i.e. independence on the choice of $\hat{g}_{0}$ in (A.27), requires

$$
C_{i j k l}=0 \quad \forall i, j, k, l .
$$

With these normalizations the vacuum diagrams vanish in the adiabatic limit

$$
\lim _{\epsilon \rightarrow 0}\left(\Omega, S_{n}\left(g_{\epsilon}\right) \Omega\right)=0 .
$$

We turn to our massless Yang-Mills model and omit the matter coupling for simplicity. In second order we have instead of (A.26)

$$
\hat{t}(p) \sim\left(p^{2}\right)^{2} \log \frac{-\left(p^{2}+i 0\right)}{M^{2}}+C_{0}+C_{2} p^{2},
$$

where the normalization term $\sim\left(p^{2}\right)^{2}$ is contained in the (arbitrary) constant $M$. Inserting this into (A.27) a divergence $\sim \log \epsilon$ cannot be avoided with any normalization. This is no harm for the Green's functions because the vacuum diagrams are absent there.

I would like to thank Frank Krahe and Prof. G.Scharf for stimulating discussions and for reading the manuscript. I am grateful to Tobias Hurth for drawing my attention to some 
of the references cited below. Finally, I thank my fiancée Annemarie Schneider for bearing with me during working at this paper.

\section{References}

[1] M.DÜTSCH, T.HURTH, F.KRAHE, G.SCHARF, N. Cimento A 106 (1993), 1029

[2] M.DÜTSCH, T.HURTH, F.KRAHE, G.SCHARF, N. Cimento A 107 (1994), 375

[3] M.DÜTSCH, T.HURTH, G.SCHARF, N. Cimento A 108 (1995), 679

[4] M.DÜTSCH, T.HURTH, G.SCHARF, N. Cimento A 108 (1995), 737

[5] M.DÜTSCH, preprint ZU-TH 10/95

[6] H.EPSTEIN, V.GLASER, Ann. Inst. Poincaré A 19 (1973), 211

[7] C.BECCHI, A.ROUET, R.STORA, Commun. Math. Phys. 42 (1975), 127

C.BECCHI, A.ROUET, R.STORA, Annals of Physics (N.Y.) 98 (1976), 287

[8] G.SCHARF, "Finite Quantum Electrodynamics", 2nd. ed., Springer-Verlag (1995)

[9] M.DÜTSCH, F.KRAHE, G.SCHARF, Nuovo Cimento A 106 (1993), 277

[10] J.C.TAYLOR, Nucl.Phys. B 33 (1971), 436

[11] A.A.SLAVNOV, Theor.a.Math.Phys. 10 (1972), 99

[12] G.'T HOOFT, Nucl. Phys. B 33 (1971), 173

[13] M.GELL-MANN, F.LOW, Phys. Rev. 84 (1951), 350

[14] M.DÜTSCH, F.KRAHE, G.SCHARF, J. Phys. G 19 (1993) 485

[15] M.DÜTSCH, F.KRAHE, G.SCHARF, J. Phys. G 19 (1993) 503

[16] P.PASCUAL, R.TARRACH, "QCD: Renormalization for the Practitioner", Springer-Verlag (1984)

[17] T.W.CHIU, Nucl.Phys. B 181 (1981), 450

[18] M.DÜTSCH, F.KRAHE, G.SCHARF, Nuovo Cimento A 103 (1990), 903

[19] S.K.KIM, M.BAKER, Nucl.Phys. B 164 (1980), 152

[20] N.N.BOGOLIUBOV, D.V.SHIRKOV, "Introduction to the Theory of Quantized Fields", New York (1959)

[21] M.DÜTSCH, F.KRAHE, G.SCHARF, Nuovo Cimento A 103 (1990), 871

[22] P.BLANCHARD, R.SENEOR, Ann. Inst. Poincaré A 23 (1975), 147

[23] T.MUTA, "Foundations of Quantum Chromodynamics", World Scientific, Singapore (1987)

\section{Figure Captions}

Fig.1. Diagrams in $x$-space representing the term $\sim g t_{A F}^{\alpha \nu \mu}\left(p_{1}, p_{2}+p_{3}, p_{4}, . ., . p_{n-1}\right)$ in (2.11). The left-handed diagram has an external four-gluon vertex at $x_{2}=x_{3}$. In causal perturbation theory this four-gluon vertex is the normalization term $-\frac{1}{2} \delta\left(x_{2}-x_{3}\right)(1.18)$ 
of the propagator $\partial^{\mu} \partial^{\nu} D_{F}\left(x_{2}-x_{3}\right)-\frac{1}{2} g^{\mu \nu} \delta\left(x_{2}-x_{3}\right)$ in the (degenerate) diagram on the r.h.s..

Fig.2. Diagramatic form of the degenerate terms contributing to the connected 3-point Green's fuctions. The terms represented by fig. 1 are excluded in fig.2, since they are absorbed in the non-degenerate terms $\sim \tilde{t}_{B_{1} B_{2} B_{3}}^{(n)}$.

Figs.3a,b,c,d. Diagramatic form of the degenerate terms contributing to the connected 4 -point Green's fuctions. Note that in higher orders the $t, \tilde{t}$-distributions contain one-particle reducible terms. We assume all external momenta $p_{i}(i \in\{1,2,3,4\})$ and all $\left(p_{i}+p_{j}\right)(1 \leq i<$ $j \leq 4)$ to be off-shell. This means that in figs.2,3a-d all momenta of the Feynman propagators and all non-vanishing momenta in the arguments of the $\tilde{t}^{(n-1)}, \tilde{t}^{(n-2)}$-distributions are offshell. 
This figure "fig1-1.png" is available in "png" format from: http://arxiv.org/ps/hep-th/9606105v1 
This figure "fig2-1.png" is available in "png" format from: http://arxiv.org/ps/hep-th/9606105v1 
This figure "fig1-2.png" is available in "png" format from: http://arxiv.org/ps/hep-th/9606105v1 
This figure "fig2-2.png" is available in "png" format from: http://arxiv.org/ps/hep-th/9606105v1 
This figure "fig1-3.png" is available in "png" format from: http://arxiv.org/ps/hep-th/9606105v1 
This figure "fig2-3.png" is available in "png" format from: http://arxiv.org/ps/hep-th/9606105v1 